\title{
KÄHLER-EINSTEIN METRICS, CANONICAL RANDOM POINT PROCESSES AND BIRATIONAL GEOMETRY
}

\author{
ROBERT J. BERMAN
}

\begin{abstract}
In the present paper and the companion paper 8 a probabilistic (statistical mechanical) approach to the study of canonical metrics and measures on a complex algebraic variety $X$ is introduced. On any such variety with positive Kodaira dimension a canonical (birationally invariant) random point processes is defined and shown to converge in probability towards a canonical deterministic measure $\mu_{X}$ on $X$, coinciding with the canonical measure of Song-Tian and Tsuji. The proof is based on new large deviation principle for Gibbs measures with singular Hamiltonians which relies on an asymptotic submean inequality in large dimensions, proved in a companion paper. In the case of a variety $X$ of general type we obtain as a corollary that the (possibly singular) Kähler-Einstein metric on $X$ with negative Ricci curvature is the limit of a canonical sequence of quasi-explicit Bergman type metrics. In the opposite setting of a Fano variety $X$ we relate the canonical point processes to a new notion of stability, that we call Gibbs stability, which admits a natural algebro-geometric formulation and which we conjecture is equivalent to the existence of a Kähler-Einstein metric on $X$ and hence to K-stability as in the Yau-Tian-Donaldson conjecture.
\end{abstract}

\section{Contents}

1. Introduction

2. Outline of the proof of the convergence and relations to statistical mechanics

3. Analytic setup

4. The LDP for $\beta$-deformed determinantal point processes

5. Canonical random point processes on varieties of positive Kodaira dimension and log pairs

6. Fano manifolds and Gibbs stability

References

\section{INTRODUCTION}

Kähler-Einstein metrics, i.e. Kähler metrics with constant Ricci curvature, play a prominent role in the study of complex algebraic varieties. When such a variety $X$ admits a Kähler-Einstein metric it is essentially unique, i.e. canonically attached to $X$ and can thus be used to probe the space $X$ using differential-geometric tools (e.g. as in Yau's proof of the Miyaoka-Yau Chern number inequalities). Singular versions of Kähler-Einstein are also naturally linked to Mori's Minimal Model Program (MMP) for complex algebraic varieties, notably through the Kähler-Ricci flow [45, 44, 48. In the case when the variety $X$ is defined over the integers, i.e. $X$ is cut 
out by polynomials with integer coefficients, it is also expected that Kähler-Einstein metrics carry arithmetic information (even if there are very few direct results in this direction). For example, the role of Kähler-Einstein metrics in arithmetic (Arakelov) geometry was speculated on by Manin [39], as playing the role of minimal models over the prime/place at infinity.

In the present paper and the companion paper 8 a new probabilistic (statisticalmechanical) approach to the study of Kähler-Einstein type metrics is introduced, where the metrics appear in the large $N$-limit of of certain canonical random point processes on $X$ with $N$ particles. The canonical point processes in question are directly defined in terms of algebro-geometric data and the thrust of this approach is thus that it gives a new direct link between algebraic geometry on one hand and complex differential (Kähler) geometry on the other. In the companion paper [8] a general convergence result for stochastic interacting particle systems in thermal equilibrium is established and applied in the setting when the canonical line bundle $K_{X}$ is positive/ample to produce the unique Kähler-Einstein metric on $X$. In the present paper the results are extended to any variety $X$ of positive Kodaira dimension, using the global pluripotential theory and variational calculus in [20, 11, 5] and some algebraic geometry (in particular the Fujino-Mori canonical bundle formula [30]). While our main results are centered around the case of negatively curved Kähler-Einstein metrics and various singular and twisted generalizations of such metrics, we also formulate a conjectural picture relating the existence problem for Kähler-Einstein metrics with positive Ricci curvature on a Fano manifold $X$ to a statistical mechanical notion of stability that we call Gibbs stability. The latter notion of stability thus replaces the notion of K-stability which appears in the seminal Yau-Tian-Donaldson conjecture for Kähler-Einstein metrics on Fano manifolds. Interestingly, the notion of Gibbs stability also admits a natural purely algebro-geometric formulation in terms of the standard singularity notions in the MMP.

The connections to physics (in particular emergent gravity and fermion-boson correspondences) were emphasized in [4], where a heuristic argument for the convergence of the point processes was outlined. In the present paper and its companion [8] we provide rigorous proofs - in a more general setting - building on [9, 10, 11, 5. The key new technical feature is a mean value inequality for quasi-subharmonic functions on Riemannian quotients in large dimensions with a distortion coefficient which is sub-exponential in the dimension proved in 8 .

Before turning to precise statements of the main results it may also be worth pointing out that there are also numerical motivations for the current approach. Indeed, there are very few examples of Kähler-Einstein metrics that can be written down explicitly and one virtue of the present framework is that it offers an, essentially, explicit way of numerically sampling random points in order to approximate the Kähler-Einstein metric. It would thus be interesting to compare it with other recently proposed numerical schemes [27, 25].

1.1. Kähler-Einstein geometry and the canonical point processes. Let $X$ be an $n$-dimensional compact complex manifold. A Kähler metric $\omega$ on $X$ is said to be Kähler-Einstein if its Ricci curvature is constant:

$$
\operatorname{Ric} \omega_{K E}=-\beta \omega_{K E}
$$


where, after normalization one may assume that $\beta= \pm 1$ or $\beta=0$. Since the Ricci form of a Kähler metric represents minus the first Chern class $c_{1}\left(K_{X}\right)$ of the canonical line bundle $K_{X}:=\Lambda^{n}(T X)$ of $X$ the Kähler-Einstein equation imposes cohomological conditions saying that $c_{1}\left(K_{X}\right)$ vanishes when $\beta=0$ and has a definite sign when $\beta= \pm 1$. In the latter case, which is the one we shall mainly focus on, this means in algebro-geometric terms that $\pm K_{X}$ is ample (using additive notation for tensor products, so that $-K_{X}$ denotes the dual of $K_{X}$ ) and in particular $X$ is a projective algebraic variety. As is well-known there are also singular versions of Kähler-Einstein metrics obtained by either relaxing the positivity (or negativity) condition on $K_{X}$ or introducing a $\log$ structure on $X$, i.e. a suitable divisor $D$ on $X$ (see below). We recall that these notions appear naturally in the Minimal Model Program, which aims at attaching a minimal model to a given algebraic variety (say with positive Kodaira dimension), i.e. a birational model whose canonical line bundle is nef (which is the numerical version of semi-positivity) [19. Recently, there has also been a rapid development of the theory of Kähler-Einstein metrics attached to a log pair $(X, D)$ (see for example [28, 40, 22, 34]).

1.1.1. Varieties of general type $(\beta=1)$. Let us start with the case when $\beta=1$, i.e. the case when $K_{X}$ is positive or more generally big (i.e. $X$ is a variety with general type). We will show how to recover the unique (singular) Kähler-Einstein metric on such a manifold $X$ from the large $N$ limit of certain canonical random point processes on $X$ with $N$ particles, defined as follows. First define the following sequence of positive integers:

$$
N_{k}:=\operatorname{dim} H^{0}\left(X, k K_{X}\right),
$$

where $H^{0}\left(X, k K_{X}\right)$ is the space of all pluricanonical (holomorphic) $n$-forms of $X$, at level $k$ (recall that we are using additive notation for tensor products). In other words, $N_{k}$ is the $k$ th plurigenus of $X$ and we recall that $X$ is said to be of general type if $N_{k}$ is of the order $k^{n}$ for $k$ large. In particular, this is the case if $K_{X}$ is ample. The starting point of the present probabilistic approach is the observation that there is a canonical probability measure $\mu^{\left(N_{k}\right)}$ on the $N_{k}$-fold product $X^{N_{k}}$ which may be defined as follows, in terms of local holomorphic coordinates $z$ on $X$ :

$$
\mu^{\left(N_{k}\right)}=:=\frac{1}{Z_{N_{k}}}\left|\left(\operatorname{det} S^{(k)}\right)\left(z_{1}, \ldots, z_{N_{k}}\right)\right|^{2 / k} d z_{1} \wedge d \bar{z}_{1} \wedge \cdots \wedge d z_{N_{k}} \wedge d \bar{z}_{N_{k}}
$$

where $d z \wedge d \bar{z}$ is a short hand for the local Euclidean volume form on $X$ determined by the local coordinates $z$ and $\operatorname{det} S^{(k)}$ is a holomorphic section of the line bundle $\left(k K_{X}\right)^{\otimes N_{k}} \rightarrow X^{N_{k}}$, defined by a generator of the determinantal line $\Lambda^{N_{k}}\left(H^{0}\left(X, k K_{X}\right)\right.$ (and thus defined up to a multiplicative complex number). Concretely, we may take $\operatorname{det} S^{(k)}$ as the following Vandermonde type determinant

$$
\left(\operatorname{det} S^{(k)}\right)\left(z_{1}, z_{2}, \ldots, z_{N}\right):=\operatorname{det}\left(s_{i}^{(k)}\left(z_{j}\right)\right),
$$

in terms of a given basis $s_{i}^{(k)}$ in $H^{0}\left(X, k K_{X}\right)$, which locally may be identified with a holomorphic function on $X^{N_{k}}$. The point is that, by the very definition of the canonical line bundle $K_{X}$, the local function $\left|\left(\operatorname{det} S^{(k)}\right)\left(z_{1}, \ldots, z_{N_{k}}\right)\right|^{2 / k}$ transforms as a density on $X^{N_{k}}$ and after normalization by its total integral $Z_{N_{k}}$ one obtains a sequence of globally well-defined probability measures $\mu^{\left(N_{k}\right)}$ on $X^{N_{k}}$ which are 
independent of the choice of basis $s_{i}^{(k)}$ in $H^{0}\left(X, k K_{X}\right)$ and thus canonically attached to $X$. From a statistical mechanical point of view the normalizing constant

$$
Z_{N_{k}}:=\int_{X^{N_{k}}}\left|\left(\operatorname{det} S^{(k)}\right)\left(z_{1}, \ldots, z_{N_{k}}\right)\right|^{2 / k} d z_{1} \wedge d \bar{z}_{1} \wedge \cdots \wedge d z_{N_{k}} \wedge d \bar{z}_{N_{k}}
$$

is the partition function and it depends on the choice of generator (but the point is that $\mu^{\left(N_{k}\right)}$ does not).

By construction the probability measure $\mu^{\left(N_{k}\right)}$ is symmetric, i.e. invariant under the natural action of the permutation group $\Sigma_{N_{k}}$ and hence defines a random point process on $X$ with $N_{k}$ particles (i.e. a probability measure on the space of all configurations of $N_{k}$ points on $X$ ). To simplify the notation we will often omit the subscript $k$ on $N_{k}$. This should cause no confusion, since $k$ tends to infinity precisely when $N_{k}$ does. We recall that the empirical measure of a random point process with $N$ particles on a space $X$ is the random measure

$$
\delta_{N}:=\frac{1}{N} \sum_{i=1}^{N} \delta_{x_{i}}
$$

on the probability space $\left(X^{N}, \mu^{(N)}\right)$ which defines a map from $X^{N}$ to the space $\mathcal{M}_{1}(X)$ of all normalized measures $\mu$ on $X$. By definition the law of $\delta_{N}$ is the pushforward of $\mu^{(N)}$ to $\mathcal{M}_{1}(X)$ under the map $\delta_{N}$, which thus defines a probability measure on the space $\mathcal{M}_{1}(X)$.

Theorem 1.1. Let $X$ be a variety of general type. Then the empirical measures of the canonical random point processes on $X$ converge in probability towards the normalized volume form $d V_{K E}$ of the Kähler-Einstein metric on X. More precisely, the laws of the empirical measures satisfy a large deviation principle with speed $N$ and rate functional $F(\mu)$, where $F\left(\omega^{n} / V\right)$ is Mabuchi's K-energy of the Kähler form $\omega$ (normalized so that $F$ vanishes on $d V_{K E}$ )

We recall that, loosely speaking, the large deviation principle referred to in the previous theorem says that the convergence in probability is exponential in the following sense:

$$
\operatorname{Prob}\left(\frac{1}{N_{k}} \sum_{i} \delta_{x_{i}} \in B_{\epsilon}(\mu)\right) \sim e^{-N F(\mu)}
$$

as first $N \rightarrow \infty$ and then $\epsilon \rightarrow 0$, where $B_{\epsilon}(\mu)$ denotes a ball of radius $\epsilon$ centered at a given probability measure $\mu$ in the space $\mathcal{M}_{1}(X)$ (equipped with a metric compatible with the weak topology). In other words this means that the probability of finding a cloud of $N$ points $x_{1}, \ldots, x_{N}$ on $X$ such that the corresponding measure $\frac{1}{N_{k}} \sum_{i} \delta_{x_{i}}$ approximates a volume form $\mu$ is exponentially small unless $\mu$ is a minimizer of $F$.

Corollary 1.2. Let $X$ be a variety of general type. Then the one-point correlation measures $\nu_{k}:=\int_{X^{N-1}} \mu^{\left(N_{k}\right)}$ of the canonical point processes define a sequence of canonical measures on $X$ converging weakly to $d V_{K E}$. Moreover, the curvature forms of the corresponding metrics on $K_{X}$ defined by the sequence $\nu_{k}$ converge weakly to the unique Kähler-Einstein metric $\omega_{K E}$ on $X$.

The last statement in the previous corollary concretely says that the unique (possible singular) Kähler-Einstein metric $\omega_{K E}$ on $X$ (whose existence was established in the seminal works of Aubin [1] and Yau [51] when $K_{X}$ is ample and in 
29, 20, 11] in general) may be recovered as the weak limit of the following sequence of quasi-explicit canonical positive currents in $c_{1}\left(K_{X}\right)$ :

$\omega_{k}:=\frac{i}{2 \pi} \partial \bar{\partial} \log \int_{X^{N_{k}-1}}\left|\left(\operatorname{det} S^{(k)}\right)\left(\cdot, z_{1}, \ldots, z_{N_{k}-1}\right)\right|^{2 / k} d z_{1} \wedge d \bar{z}_{1} \wedge \cdots \wedge d z_{N_{k}-1} \wedge d \bar{z}_{N_{k}-1}$

which are smooth away from the base locus of $k K_{X}$. It may be worthwhile pointing out that it does not seem clear how to prove the convergence of the one-point correlation measures without first proving the full large deviation principle in the previous theorem, which in general is a considerably stronger type of convergence result.

The convergence of the currents $\omega_{k}$ towards $\omega_{K E}$ is somewhat analogous to Donaldson's convergence of balanced metrics towards the constant scalar curvature metric attached to a polarized manifold $(X, L)$ with finite automorphism group [26]. Indeed, in both situations the approximating metrics in question are singled our by the sequence of finite dimensional spaces $H^{0}(X, k L)$. One virtue of the present setting is that it applies when $L\left(=K_{X}\right)$ is merely big thus providing what seems to be the first general approximation result for the singular Kähler-Einstein metric $\omega_{K E}$ on a variety $X$ of general type.

1.1.2. Birational invariance and varieties of positive Kodaira dimension. The results for varieties of general type above generalize to the setting of a projective variety $X$ of positive Kodaira dimension $\kappa$. The starting point is the observation that the canonical random point processes introduced above are well-defined as long as the plurigenera $N_{k}$ are non-zero and that they are invariant under birational equivalence of varieties. In order to do asymptotics we also need $N_{k}$ to tend to infinity, which means that the natural setting for the canonical point processes is a projective variety $X$ of positive Kodaira dimension $\kappa$ (recall that $\kappa$ is the natural number defined by the growth property $N_{k} \sim k^{\kappa}$ ).

Theorem 1.3. Let $X$ be a projective variety of positive Kodaira dimension. Then the empirical measures of the canonical random point processes on $X$ converge in probability towards a unique probability measure $\mu_{X}$, which coincides with the canonical measure of Song-Tian and Tsuji.

The canonical measure $\mu_{X}$ was introduced by Song-Tian [45] in their study of the Kähler-Ricci flow and, independently, by Tsuji [48] in his study of dynamical systems defined by Bergman kernels. The measure $\mu_{X}$ is defined in terms of the Iitaka fibration $F: X \rightarrow Y$ attached to $X:$ on the Zariski open subset $Y_{0}$ where $F$ defines a smooth morphism the measure $\mu_{X}$ may be written as

$$
\mu_{X}=\left(F^{*} \omega_{Y}\right)^{\kappa} \wedge\left(\omega_{C Y}\right)^{n-\kappa},
$$

where, fiberwise, $\omega_{C Y}$ is a Ricci flat metric with normalized volume and $\omega_{Y}$ is a canonical metric defined on the base $Y$ in terms of a twisted Kähler-Einstein equation: it satisfies (in a weak sense) the equation

$$
\operatorname{Ric} \omega_{Y}=-\omega_{Y}+\omega_{W P}
$$

away from the branch locus of the fibration, where $\omega_{W P}$ is the generalized WeilPetersson metric on $Y$, measuring the infinitesimal variation of the complex moduli of the Calabi-Yau fibers (see section 5.2 for precise definitions). The proof of the 
previous theorem relies on Fujino-Mori's canonical bundle formula [30] which allows one to reduce the problem to the base $Y$ of the Iitaka fibration. A similar argument was used by Tsuji in a different context [48. It was shown by Song-Tian [45] that, in the case when $K_{X}$ is semi-ample, the Kähler-Ricci flow on $X$ converges weakly towards a canonical positive current which coincides with $F^{*} \omega_{Y}$. In our setting we obtain as an immediate corollary of the previous theorem that, for any variety of positive Kodaira dimension, the canonical sequence of currents $\omega_{k} \in c_{1}\left(K_{X}\right)$ defined by formula 1.5 converges weakly to a canonical positive current in $c_{1}\left(K_{X}\right)$ with minimal singularities, coinciding with $F^{*} \omega_{Y}$ on a Zariski open subset of $X$. This is hence more general then assuming that $K_{X}$ be semi-ample. On the other hand, assuming the validity of the fundamental conjectures of the MMP, i.e. the existence of a minimal model and the abundance conjecture (which is not necessary for our approach) the convergence of $\omega_{k}$ reduces to the case when $K_{X}$ is semi-ample.

1.1.3. Logarithmic generalizations and twisted Kähler-Einstein metrics. The results stated above admit natural generalization to the logarithmic setting, in the sense of MMP, which from the differential geometric point of view is related to KählerEinstein metrics with conical and cusp type singularities. To explain this first recall that (in the smooth setting) a log canonical pair $(X, D)$ consists of a smooth complex algebraic variety $X$ and a $\mathbb{Q}$-divisor $D$ on $X$ with simple normal crossings and coefficients in $]-\infty, 1]$. In this setting the role of the canonical line bundle $K_{X}$ is placed by the log canonical line bundle $K_{X}+D$ and the role of the Ricci curvature Ric $\omega$ of a metric $\omega$ is played by twisted Ricci curvature Ric $\omega-[D]$, where $[D]$ denotes the current of integration defined by $D$. The corresponding (twisted) Kähler-Einstein equation thus reads

$$
\text { Ric } \omega=\beta \omega+[D]
$$

which should be interpreted in a weak sense (see [14 for a very general setting). In case the coefficients of $D$ are in $[0,1$ [ the pair $(X, D)$ is said to be Kawamata log terminal ( $k l t$, for short) and if $K_{X}+D$ is ample the solution of the corresponding (twisted) Kähler-Einstein equation then has conical singularities along $D$ [28, 40, 22, 34].

To any klt log pair $(X, D)$ we may attach a sequence of canonical probability measures $\mu^{\left(N_{k}\right)}$ on $X^{N_{k}}$ defined as follows. We take $\operatorname{det} S^{(k)}$ to be a generator in the determinant line of $H^{0}\left(X, k\left(K_{X}+D\right)\right)$ and multiply the local volume forms $d z \wedge d \bar{z}$ with $1 /\left|s_{D}\right|^{2}$, where $s_{D}$ is a global holomorphic (multivalued) section cutting out $D$. The proof of the corresponding convergence results can then be deduced from the general Theorem 4.11, using that the pair $(X, D)$ determines a finite measure $\mu_{0}$ which is absolutely continuous with respect to the Lebesgue measure (with an $L^{p}$-density, for some $p>1$ ) whose singularities/degeneracies are encoded by the divisor $D$.

More generally, as will be shown elsewhere, the convergence results for klt pairs can be extended to the setting of log canonical pairs $(X, D)$. In the particular case when the the pair $(X, D)$ is defined over the integers the present probabilistic framework then turns out to be connected to the theory of cusp forms and canonical heights in Arakelov geometry. In a nutshell, the point is that when $(X, D)$ is defined over the integers the corresponding generator $\operatorname{det} S^{(k)}$ can be taken to be defined over the integers and since such a generator is uniquely determined up to a sign the corresponding partition function $Z_{k}$ is canonically attached to the 
integral structure. Using the generalization of Gillet-Soulé's arithmetic HilbertSamuel theorem in [13] it can then be shown that $-\frac{1}{N_{k}} \log Z_{N_{k}}$ converges, as $k \rightarrow \infty$, to the height (i.e. the top arithmetic intersection number) of the corresponding integral model of $K_{X}+D$, with respect to the Kähler-Einstein metric.

Acknowledgment. It is a pleasure to thank Sebastien Boucksom, David Witt-Nyström, Vincent Guedj and Ahmed Zeriahi for the stimulating collaborations [9, 10, 11], which paved the way for the present work. I am also grateful to Bo Berndtsson for infinitely many fruitful discussions on complex analysis and Kähler geometry over the years. The present paper, together with the companion paper [8, supersedes the first arXiv version of the paper (the latter paper also contained results about log pairs with log canonical singularities and applications to arithmetic geometry, which will appear in a separate paper). This work was supported by grants from the ERC and the KAW foundation

Organization. To make the paper reasonably self-contained we start in Section 2 by outlining the proof of the LDP in Theorem 1.1 and point out some relations to statistical mechanics which are the subject of the companion paper [8]. Then in Section 3 we setup the analytical/pluripotential framework needed for the actual proofs of the main results. Since the paper has been written mainly for readers with a background in complex geometry in mind we start Section 4 with a lightning review of the relevant notions in probability theory (that can be found in any standard probability text book). Then the proof of a general LDP for certain $\beta$-deformations of determinantal point processes is given (using the asymptotic submean inequality proved in the companion paper [8], where a general LDP for Gibbs measures is also proved. In Section 5.2 it is shown how to apply the general LDP in the complex geometric framework of canonical point processes of klt pairs and varieties of of positive Kodaira dimension to prove the results formulated in the introduction of the paper. Finally, in Section 6 we show how to define canonical point processes on Fano manifolds and discuss the relations to a new notion of stability, stating a number of conjectures.

\section{Outline of the PROOF of the CONVERGEnCE AND RElations to STATISTICAL MECHANICS}

In this section we outline the proof of the large deviation principle in Theorem 5.1 concerning the case when $X$ is a variety of general type, i.e. the canonical line bundle $K_{X}$ is big. In fact the same arguments apply to the more general setting of $\beta$-deformed determinantal point processes associated to a big line bundle $L$ described in Section 4 .

Fixing a reference volume form $d V$ on $X$ and denoting by $\|\cdot\|$ the corresponding metric on the canonical line bundle $L:=K_{X}$ the canonical probability measure $\mu^{\left(N_{k}\right)}$ on $X^{N}$ can be factorized as

$$
\mu^{\left(N_{k}\right)}=\frac{1}{Z_{N_{k}}}\left\|\operatorname{det} S^{(k)}\right\|^{2 / k} d V^{\otimes N_{k}},
$$

in terms of the induced metric on $K_{X^{N_{k}}}$ for any given generator $\operatorname{det} S^{(k)}$ of the determinant line $\operatorname{det} H^{0}\left(X, k K_{X}\right)$. It will be convenient to take $\operatorname{det} S^{(k)}$ to be the generator determined by a basis in $H^{0}(X, k L)$ which is orthonormal with respect to the $L^{2}$-product determined by $(\|\cdot\|, d V)$ for any fixed volume form $d V$ on $X$. By 
general principles (Lemma 4.7) it will be enough to obtain the corresponding LDP for the non-normalized measures on $X^{N_{k}}$ obtained by discarding the normalization factor $Z_{N_{k}}$. Fixing a metric on the space $\mathcal{M}_{1}(X)$ of all normalized measures $\mu$ on $X$, compatible with the weak topology, it will be convenient to identify a ball $B_{\epsilon}(\mu)$ in $\mathcal{M}_{1}(X)$, centered at $\mu$ of radius $\epsilon$, with a subset of $X^{N}$ using the map

$$
\delta_{N}:=\frac{1}{N} \sum_{i=1}^{N} \delta_{x_{i}}: X^{N} \rightarrow \mathcal{M}_{1}(X) .
$$

Then the large deviation principle (LDP) in Theorem 5.1, is equivalent to the following upper and lower bounds on the integral of $\mu^{\left(N_{k}\right)}$ over small balls $B_{\epsilon}(\mu)$ :

$$
\lim _{\epsilon \rightarrow 0} \limsup _{k \rightarrow \infty} \frac{1}{N_{k}} \log \int_{B_{\epsilon}(\mu)}\left\|\operatorname{det} S^{(k)}\right\|^{2 / k} d V^{\otimes N_{k}} \leq-F(\mu)
$$

and

$$
\lim _{\epsilon \rightarrow 0} \liminf \frac{1}{N_{k}} \log \int_{B_{\epsilon}(\mu)}\left\|\operatorname{det} S^{(k)}\right\|^{2 / k} d V^{\otimes N_{k}} \geq-F(\mu),
$$

for a functional $F$ on $\mathcal{M}_{1}(X)$ called the rate functional of the LDP (in the present setting this could be taken as the definition of the LDP, but see Section 4.1 for the general situation). The idea of the proof of the LDP is to handle the the density $\left\|\operatorname{det} S^{(k)}\right\|^{2 / k}$ and the reference measure $d V^{\otimes N_{k}}$ separately. The point is that, if the density would have been constant, i.e. if $\mu^{(N)}=d V^{\otimes N}$, a classical theorem of Sanov says that the LDP above holds with rate function given by the entropy of $\mu$ relative to $\mu_{0}$, i.e. the functional defined by

$$
D_{\mu_{0}}(\mu):=\int_{X} \log \frac{\mu}{\mu_{0}} \mu,
$$

when $\mu$ has a density with respect to $\mu_{0}$ and otherwise $D_{\mu_{0}}(\mu):=\infty$. To handle the present setting we will combine Sanov's theorem with the logarithmic asymptotics for the sup of $\left\|\operatorname{det} S^{(k)}\right\|^{2}$ established in [9]: for any given any function $u \in C^{0}(X)$ $(2.2)$

$$
\lim _{k \rightarrow \infty} \frac{1}{k N_{k}} \log \sup _{X^{N_{k}}}\left(\left\|\operatorname{det} S^{(k)}\right\|^{2}\left(x_{1}, \ldots, x_{N_{k}}\right) e^{-k u\left(x_{1}\right)+\ldots+k u\left(x_{N_{k}}\right)}\right)=-\mathcal{F}(u),
$$

where $\mathcal{F}$ is a certain functional on the space $C^{0}(X)$ whose definition we will come back to below (in geometric terms the factor involving $u$ just corresponds to replacing the background metric $\|\cdot\|$ with $\left.\|\cdot\|_{u}:=\|\cdot\| e^{-u / 2}\right)$.

2.1. The upper bound in the LDP. To prove the upper bound of the integrals appearing in the LDP 2.1 we fix a function $u \in C_{0}(X)$ and rewrite

$$
\left\|\operatorname{det} S^{(k)}\right\|^{2 / k}=\left(\left\|\operatorname{det} S^{(k)}\right\|^{2} e^{-k u}\right)^{1 / k} e^{u},
$$

where we have identified $u$ with the corresponding function $u\left(x_{1}\right)+\ldots+u\left(x_{N_{k}}\right)$ on $X^{N}$. Then, trivially, for any fixed $\epsilon>0$,

$$
\int_{B_{\epsilon}(\mu)}\left\|\operatorname{det} S^{(k)}\right\|^{2 / k} d V^{\otimes N_{k}} \leq \sup _{B_{\epsilon}(\mu)}\left(\left\|\operatorname{det} S^{(k)}\right\|^{2} e^{-k u}\right)^{1 / k} \int_{X^{N}} \mu_{u}^{\otimes N_{k}}, \mu_{u}:=e^{u} d V
$$


Hence, replacing the sup over $B_{\epsilon}(\mu)$ with the sup over all of $X^{N_{k}}$ and applying Sanov's theorem relative to the tilted volume form $\mu_{u}$ gives

$$
\lim _{\epsilon \rightarrow 0} \limsup _{k \rightarrow \infty} \frac{1}{N_{k}} \log \int_{B_{\epsilon}(\mu)}\left\|\operatorname{det} S^{(k)}\right\|^{2 / k} d V^{\otimes N_{k}} \leq-\mathcal{F}(u)-\int_{X} u \mu-D_{d V}(\mu),
$$

using that $D_{e^{u} d V}(\mu)=-\int u \mu+D_{d V}(\mu)$. Finally, taking the infimum over all $u \in C^{0}(X)$ gives an upper bound on the limsup appearing in formula 2.1 with $F(\mu)$ defined by

$$
F(\mu):=\mathcal{F}^{*}(\mu)+D_{d V}(\mu), \quad \mathcal{F}^{*}(\mu):=\sup _{u \in C^{0}(X)}\left(\int_{X} u \mu+\mathcal{F}(u)\right)
$$

2.2. The lower bound in the LDP. As usually the proof of the lower bound in the LDP is the hardest one. The starting point is the fact, proved in [9], that the limiting functional $\mathcal{F}$ on $C^{0}(X)$ appearing in formula2.2 is Gateaux differentiable on $C^{0}(X)$ (it is also concave, as follows directly from its definition) and its differential at a given $u \in C^{0}(X)$ is represented by a probability measure $d \mathcal{F}_{\mid u}$. In particular, the sup in the formula defining $\mathcal{F}^{*}(\mu)$ is attained iff there exists a function $u_{\mu} \in C^{0}(X)$ such that

$$
d \mathcal{F}_{\mid u_{\mu}}=\mu \text {. }
$$

Assuming that the measure $\mu$ appearing in the LDP has this property the idea of the proof of the LDP is to try to reverse the trivial inequality 2.3 for $u=u_{\mu}$. To this end we first recall that, as observed in [10, it follows from a simple convex analysis argument that, if $\mathbf{x}^{\left(N_{k}\right)}$ in $X^{N_{k}}$ denotes a sequence of ordered configurations of points realizing the sup in formula 2.2 for a given $u$, then

$$
\delta_{N}\left(\mathbf{x}^{\left(N_{k}\right)}\right) \rightarrow d \mathcal{F}_{\mid u}
$$

in $\mathcal{P}(X)$, with respect to the weak topology. In particular, taking $u=u_{\mu}$ it follows that, for any fixed $\epsilon>0$,

$$
B_{\epsilon / 2}(\mu) \subset B_{\epsilon}\left(\delta\left(\mathbf{x}^{\left(N_{k}\right)}\right)\right) \subset B_{2 \epsilon}(\mu)
$$

for $N$ sufficiently large. Accordingly,

$$
\int_{B_{2 \epsilon}(\mu)}\left\|\operatorname{det} S^{(k)}\right\|^{2 / k} d V^{\otimes N_{k}} \geq \int_{B_{\epsilon}\left(\delta\left(\mathbf{x}^{\left(N_{k}\right)}\right)\right)}\left\|\operatorname{det} S^{(k)}\right\|_{u}^{2 / k} \mu_{u}^{\otimes N}
$$

and hence it will be enough to establish an asymptotic submean property of the following form

$$
\int_{B_{\epsilon}\left(\delta\left(\mathbf{x}^{\left(N_{k}\right)}\right)\right)}\left\|\operatorname{det} S^{(k)}\right\|_{u}^{2 / k} \mu_{u}^{\otimes N} \geq e^{N \epsilon^{\prime}}\left\|\operatorname{det} S^{(k)}\left(\mathbf{x}^{\left(N_{k}\right)}\right)\right\|_{u}^{2 / k} \int_{B_{\epsilon^{\prime \prime}}\left(\delta\left(\mathbf{x}^{\left(N_{k}\right)}\right)\right)} \mu_{u}^{\otimes N}
$$

for some positive numbers $\epsilon^{\prime}$ and $\epsilon^{\prime \prime}$ smaller than $\epsilon$. Indeed, we would then get

$\liminf _{k \rightarrow \infty} \frac{1}{N_{k}} \log \int_{B_{2 \epsilon}(\mu)}\left\|\operatorname{det} S^{(k)}\right\|^{2 / k} d V^{\otimes N_{k}} \geq-\epsilon^{\prime}-\mathcal{F}\left(u_{\mu}\right)+\liminf _{k \rightarrow \infty} \frac{1}{N_{k}} \log \int_{B_{\epsilon^{\prime \prime} / 2}(\mu)} \mu_{u}^{\otimes N}$

and letting $\epsilon \rightarrow 0$ we could then conclude the proof by invoking Sanov's theorem again. We recall that we made the assumption 2.4 that the fixed measure $\mu$ be contained in the image of $C^{0}(X)$ under the "gradient map" $d \mathcal{F}$. But in fact all that is needed in the proof of the lower bound in the LDP given above is that any $\mu$ 
such that $\mathcal{F}^{*}(\mu)<\infty$ has the following "regularization property": there exists a sequence of $u_{j} \in C^{0}(X)$ such that

$$
\mu_{j}:=d \mathcal{F}_{\mid u_{j}} \rightarrow \mu, \quad \mathcal{F}^{*}\left(\mu_{j}\right) \rightarrow \mathcal{F}^{*}(\mu)
$$

In the present setting this property is shown in Lemma 3.7 (but it can also be obtained from general convex analysis results [8]).

As for the submean property 2.5] it is proved in the companion paper [8, with $\epsilon^{\prime}=\epsilon$ and $\epsilon^{\prime \prime}=\epsilon^{2}$ (the result also appeared as Theorem 2.1 in the first preprint version of the present paper on ArXiv). The starting point of the proof is the observation that if the metric on $\mathcal{M}_{1}(X)$ is taken as the $L^{2}$-Wasserstein metric induced by a fixed Kähler metric on $X$ with volume form $d V$, then $B_{\epsilon}\left(\delta\left(\mathbf{x}^{\left(N_{k}\right)}\right)\right)$ may be identified with the pullback to $X^{N}$ of the ball of radius $\epsilon$ centered at the projection of the point $\mathbf{x}^{\left(N_{k}\right)}$ in the Riemannian orbifold $X^{(N)}:=X^{N} / S^{N}$, where $S^{N}$ denotes the symmetric group with $N$ elements acting by permutations on $X^{N}$. The inequality in question then follows from a new submean inequality for quasi-subharmonic functions on Riemannian orbifold quotients in large dimensions, obtained as refinement of a Riemannian submean inequality of Li-Schoen [36] (using, in particular, the explicit dependence on the dimension in the Cheng-Yau gradient estimate on Riemannian manifolds).

2.3. Identification of the rate function with the K-energy. It follows from general principles that the rate functional $F(\mu)$ is lower semi-continuous (lsc) and strictly convex on $\mathcal{M}_{1}(X)$ (using the strict convexity of the relative entropy). In particular, it admits a unique minimizer $\mu_{\min }$. To see that, in the present setting, $\mu_{\min }$ is the volume form of the Kähler-Einstein metric, one needs to invoke the explicit expression for the differential $d \mathcal{F}$ obtained in [9]:

$$
d \mathcal{F}_{\mid u}=\frac{1}{V}\left(\theta+d d^{c}\left(P_{\theta} u\right)^{n}, \quad d d^{c}:=\frac{i}{2 \pi} \partial \bar{\partial}, V:=K_{X}^{n}\right.
$$

where $\theta$ is the curvature form of the reference metric $\|\cdot\|$ on $K_{X}$ and $P_{\theta}$ is the maximally increasing projection operator from $C^{0}(X)$ to the space $P S H(X, \theta)$ of all $\theta$-plurisubharmonic (psh) functions on $X$ and where the complex Monge-Ampère operator $\left(\theta+d d^{c} \varphi\right)^{n}$ on $P S H(X, \theta)$ is defined in the sense of pluripotential theory (as recalled in Section 3). In case $K_{X}$ is ample one can then invoke the Aubin-Yau theorem giving the existence of a Kähler-Einstein metric $\omega_{K E}$ on $X$ and directly check that its volume form $\mu_{K E}$ is a critical point of $\mathcal{F}$ and hence, by convexity, $\mu_{K E}=\mu_{\min }$ (see [8]). Alternatively, under the Calabi-Yau isomorphism

$$
\omega \mapsto \omega^{n} / V
$$

between the space $\mathcal{K}\left(K_{X}\right)$ of all Kähler metrics in $K_{X}$ and the subspace of $\mathcal{M}_{1}(X)$ consisting of volume forms the rate functional $F$ gets identified (on the dense subset of volume forms) with Mabuchi's K-energy functional $\kappa$ whose unique critical point in $\mathcal{K}\left(K_{X}\right)$ is the Kähler-Einstein metric $\omega_{K E}$ (see Section 4.3). One virtue of the functional $F$ is that it is defined on the whole space $\mathcal{M}_{1}(X)$ and can thus be used as an extension of the K-energy to the space of all metrics of finite energy [5, 12] (as further exploited in [15] to study a weak version of the Calabi flow realized as a weak gradient flow of the extended K-energy functional, following [43]).

In the general case of a variety $X$ of general type the existence of a unique Kähler-Einstein current $\omega_{K E}$ in $c_{1}\left(K_{X}\right)$ with minimal singularities was established 
in [20] (using various approximations to reduce the problem to the Aubin-Yau theorem on a sequence of blow-ups of $X$ ) and in [11 by a direct variational approach. The current $\omega_{K E}$ can also be obtained as a consequence of the results in [29] concerning Kähler-Einstein metrics on canonically polarized varieties with canonical singularities, using the deep finite generation of the canonical ring [19].

2.4. Relations to statistical mechanics and Gibbs measures. Next, we briefly point out some relations between the present setting and statistical mechanics (which are further expanded on in [8]). Consider a system of $N$ identical particles on a space $X$ interacting by the microscopic interaction energy $H^{(N)}\left(x_{1}, \ldots, x_{N}\right)$ on $X^{N}$ assumed symmetric (as the particles are identical) - the function $H^{(N)}$ is often called the Hamiltonian. Given a reference volume form $\mu_{0}$ on $X$ the distribution of particles, in thermal equilibrium at the inverse temperature $\beta \in \mathbb{R}$, is described by the random point process with $N$ particles defined by the corresponding Gibbs measure:

$$
\mu_{\beta}^{(N)}:=e^{-\beta H^{(N)}} \mu_{0}^{\otimes N} / Z_{N, \beta}, \quad Z_{N . \beta}:=\int_{X^{N}} e^{-\beta_{N} H^{(N)}} \mu_{0}^{\otimes N},
$$

where the normalizing constant $Z_{N, \beta}$ is called the partition function and is assumed to be finite. Formally, in the zero temperature limit $\beta=\infty$ the corresponding empirical measure $\delta_{N}$ is thus concentrated on the configurations of points $\mathbf{x}^{(N)}:=$ $\left(x_{1}, \ldots, x_{N}\right)$ minimizing $H^{(N)}$ on $X^{N}$. If the weak limit

$$
\lim _{N \rightarrow \infty} \delta_{N}\left(\mathbf{x}^{(N)}\right):=\mu_{\infty}
$$

exists in $\mathcal{M}_{1}(X)$ we can thus think of $\mu_{\infty}$ as representing the deterministic macroscopic equilibrium state of the system at zero temperature (which is independent of the reference measure $\mu_{0}$ on $X$ ). In the opposite case of infinite temperature, i.e. $\beta=0$ the system is completely random at the microscopic level, i.e. the positions $x_{i}$ are independent random points in $X$ with identical distribution $\mu_{0}$. However, macroscopically (i.e. as $N \rightarrow \infty$ ) the corresponding random measures $\delta_{N}$ converge, by Sanov's theorem, exponentially in probability towards the deterministic measure $\mu_{0}$ at a rate $N$ with rate function given by the entropy $D_{\mu_{0}}(\mu)$ relative to $\mu_{0}$. 11 But the question is what happens in at a fixed finite positive inverse temperature $\beta$ ? According, to some time-honored heuristics in thermodynamics the corresponding macroscopic measure $\mu_{\beta}$ should minimize the corresponding free energy functional $F_{\beta}$ on $\mathcal{M}_{1}(X)$ :

$$
F_{\beta}(\mu):=E(\mu)+\frac{1}{\beta} D_{\mu_{0}}(\mu)
$$

where $E(\mu)$ is a macroscopic analog of the microscopic interaction energy $H^{(N)}$, which is minimized on the zero temperature state $\mu_{\infty}$. The argument outlined in the previous section (and proved in the companion paper $[8]$ in full generality) shows that these heuristic can be made precise, in the sense of large deviations, under the following assumptions on $H^{(N)}$ (assuming for simplicity that $X$ is compact):

\footnotetext{
${ }^{1}$ The entropy is usually defined as $-D_{\mu_{0}}(\mu)$ in the physics literature, denoted by $S$, and Sanov's theorem can be seen as a mathematical justification of Boltzmann's original formula expressing the entropy as the logarithm of the number of microscopic states consistent with a given macroscopic state.
} 
- The limit of linearly perturbed minimal energies

$$
\mathcal{F}(u):=\lim _{N \rightarrow \infty} \inf _{X^{N}} \frac{1}{N}\left(H^{(N)}+u\right)
$$

exists, for any $u \in C^{0}(X)$ and the functional $\mathcal{F}$ is Gateux differentiable on $C^{0}(X)$ (with a differential $d \mathcal{F}$ taking values in $\mathcal{M}_{1}(X)$ )

- The interaction energy $H^{(N)}$ is uniformly quasi-superharmonic, i.e. there exists a constant $C>0$ such that

$$
\Delta_{x_{1}} H^{(N)} \leq C,
$$

where $\Delta$ denotes the Laplacian defined with respect to a fixed Riemannian metric on $X$ and the subscript $x_{1}$ indicates that the Laplacian acts on the first variable.

Then the macroscopic energy functional $E(\mu)$ is defined as the Legendre-Fenchel transform of the functional $\mathcal{F}(u)$, and the LDP shown in the companion paper [8] says that the laws of the corresponding empirical measures $\delta_{N}$ satisfy a LDP with rate functional $F_{\beta}$ at a speed $\beta N$. Moreover, under suitable regularity assumptions the unique minimizer $\mu_{\beta}$ of $F_{\beta}$ can be represented as $\mu_{\beta}=d \mathcal{F}_{\mid u_{\beta}}$ for a function $u_{\beta}$ satisfying the following equation:

$$
d \mathcal{F}_{\mid u_{\beta}}=e^{\beta u_{\beta}}
$$

However, unless $H^{(N)}$ is uniformly equicontinuous the function $u_{\beta}$ will, in general, not be continuous. For example, in the present setting $u_{\beta}$ will be a $\theta-$ psh function (of finite energy).

The general LDP above generalizes (in the case $\beta>0$ ) the mean field type results in [21, 35] concerning the vortex model for turbulence in two real dimensions (which in turn extend to a singular setting previous results in [38] ). In the latter setting the Hamiltonian $H^{(N)}$ is of the explicit form

$$
H^{(N)}\left(x_{1}, \ldots, x_{N}\right)=-\frac{1}{(N-1)} \sum_{1 \leq i<j \leq N} G\left(x_{i}, x_{j}\right)
$$

for a symmetric function $g$ (independent of $N$ ) - in fact, $G$ is the Green function of the corresponding Laplacian, which is thus singular along the diagonal. However, while the analysis in [21, 35] reduces, thanks to the explicit formula above, to properties of the function $G$, the main point of the present approach is that it applies in situations where $H^{(N)}\left(x_{1}, \ldots, x_{N}\right)$ does not admit any tractable explicit formula, as in the complex geometric setting. In fact, a tractable formula does exist in one complex dimension, namely the so called bosonization formula on a Riemann surface which involves explicit theta functions and regularized determinants of Laplacians (the formula was used in a related large deviation setting in [50]). The bosonization formula is particularly useful in the case $\beta<0$ on a Riemann surface as will be explained in a separate publication.

\section{Analytic Setup}

In this section we introduce the analytical framework from [9, 11] needed for the proofs of the main results stated in the introduction of the paper. As a courtesy to the reader we also sketch the proofs of the relevant results in [9, 11].

3.1. Setup. Let $L \rightarrow X$ be a holomorphic line bundle over an $n$-dimensional compact complex manifold $X$. 
3.1.1. Metrics, weights and $\theta-$ psh functions. We will denote by $\|\cdot\|$ a Hermitian metric on $L$. Occasionally we will use additive notation for metrics, where the metric is represented by a weight denoted by $\phi$, which is a short hand for a collection of local functions: if $s$ is a trivializing local holomorphic section of $L$, i.e. $s$ is nonvanishing an a given open set $U$ in $X$, then $\phi_{\mid U}:=\log \|s\|^{2}$. In this notation the normalized curvature form of $\|\cdot\|$ may be (locally) written as

$$
\theta:=d d^{c} \phi, \quad d d^{c}:=\frac{i}{2 \pi} \partial \bar{\partial},
$$

representing, globally, the first Chern class $c_{1}(L)$ in the cohomology group $H^{2}(X, \mathbb{R}) \cap$ $H^{2}(X, \mathbb{Z})$. More generally, if the weight $\phi$ is in $L_{l o c}^{1}$, the previous formula defines the curvature as a current on $X$. A (possibly singular( metric is said to be of positive curvature if $\theta \geq 0$ holds in the sense of currents. Following standard practice it will also be convenient to identify positively curved metrics on $L$ with $\theta$-psh functions, as follows. Fixing once and for all a smooth Hermitian metric $\|\cdot\|$ on $L$ any other continuous metric may be written as

$$
\|\cdot\|_{\varphi}^{2}:=e^{-\varphi}\|\cdot\|^{2}
$$

for a continuous function $\varphi$ on $X$, i.e. $\varphi \in C^{0}(X)$. In particular, the curvature current of the metric $\|\cdot\|_{\varphi}^{2}$ may then be written as

$$
\theta_{\varphi}:=\theta+d d^{c} \varphi
$$

More generally, this procedure gives a correspondence between the space of all (singular) metrics on $L$ with positive curvature current and the space $\operatorname{PSH}(X, \theta)$ defined as the space of of all upper-semi continuous functions $\varphi$ on $X$ such that $\theta_{\varphi} \geq$ 0 holds in the sense of currents. We will use the same notation $\|\cdot\|$ for the induced metrics on tensor powers of $L$ etc. In the weight notation the induced weight on the $k$ th tensor power $k L$ is thus given by $k \phi$. We recall that $\varphi_{1} \in P S H(X, \theta)$ is said to be more singular than $\varphi_{2} \in P S H(X, \theta)$ if there exists a constant $C$ such that $\varphi_{1} \leq \varphi_{2}+C$.

Example 3.1. A volume form $d V$ on $X$ induces a smooth metric on $L:=K_{X}:=$ $\operatorname{det}\left(T^{*} X\right)$ whose local weight is given by $\phi_{U}:=\log \frac{d V}{\frac{i}{2} d z_{1} \wedge d \bar{z}_{1} \wedge \cdots}$ in the local trivialization $\frac{i}{2} d z_{1} \wedge d \bar{z}_{1} \wedge \cdots \wedge \frac{i}{2} d z_{n} \wedge d \bar{z}_{n}$ of $K_{X}$ induced by given local holomorphic coordinates $z_{1}, \ldots, z_{n}$ on $U \subset X$. If $d V$ is the volume form of a Kähler metric $\omega$ on $X$, then the curvature form $\theta$ of the corresponding metric on $K_{X}$ coincides with - Ric $\omega$.

3.1.2. Holomorphic sections and big line bundles. We will denote by $H^{0}(X, k L)$ the space of all global holomorphic sections with values in the $k$ th tensor power of $L$. We will usually assume that $L$ is $b i g$, i.e.

$$
N_{k}:=\operatorname{dim} H^{0}(X, k L)=V k^{n}+o\left(k^{n}\right),
$$

for a positive number $V$, called the volume of $L$. In the case when $L=K_{X}$ and $s_{k} \in H^{0}\left(X, k K_{X}\right)$ we can attach a canonical measure $\mu_{s_{k}}$ on $X$ to $s_{k}$ which we will, abusing notation somewhat, sometimes write as

$$
\mu_{s_{k}}:=i^{n^{2}}\left(s_{k} \wedge \overline{s_{k}}\right)^{1 / k}=\left|s_{k}\right|^{2 / k} i d z \wedge d \bar{z},
$$

where in the right hand side we have locally identified $s_{k}$ with a holomorphic function, defined with respect to the local trivialization $d z^{\otimes k}$ of $K_{X}\left(d z:=d z_{1} \wedge \cdots \wedge d z_{n}\right.$ ) induced by a choice of local coordinates $z$. 
3.1.3. Divisors, log pairs and singular volume forms. We recall that an $(\mathbb{R}-)$ divisor $D$ on a complex manifold $X$ is a formal finite sum of one-dimensional irreducible subvarieties:

$$
D=\sum_{i=1}^{m} c_{i} D_{i}, \quad c_{i} \in \mathbb{R}
$$

Following the standard notation in Minimal Model Program (MMP) a pair $(X, D)$ is called a log pair and $(X, D)$ it is said to be $\log$ smooth if $D$ has simple normal crossings (snc), i.e. locally we can always choose holomorphic coordinates so that $\left.D_{i}=\left\{z_{i}=0\right\}\right)$. Henceforth $(X, D)$ will always refer to a log smooth pair (anyway this can always be arranged by passing to a log resolution). The pair $(X, D)$ is said to be $\log$ canonical (lc) if $c_{i} \leq 1$ and Kawamata Log Terminal (klt) if $c_{i}<1$. Denoting by $s_{i}$ a holomorphic section of the line bundle $\mathcal{O}\left(D_{i}\right)$ cutting out $D_{i}$ we will use the symbolic notation $s_{D}:=s_{1}^{c_{1}} \cdots s_{m}^{c_{m}}$ (which can viewed as a multi-section when $c_{i} \in \mathbb{Q}$ ). The point is that $\phi_{D}:=\log \left|s_{D}\right|^{2}$ is then a well-defined weight on the corresponding $\mathbb{R}$-line bundle $\mathcal{O}(D)$ and its curvature current coincides with the integration current $[D]$ defined by the divisor $D$. Given a volume form $d V$ on $X$ and a continuous metric on the $\mathbb{R}$-line bundle $\mathcal{O}(D)$ we obtain a measure

$$
\mu=\left\|s_{D}\right\|^{-2} d V
$$

on $X$, which thus has zeroes and poles along the irreducible divisors $D_{i}$ with negative and positive coefficients, respectively The klt assumption is equivalent to demanding that $\mu$ be a finite measure. We will say that $\mu$ defined as above is a singular volume form in the singularity class defined by $D$.

3.2. Preliminaries on complex Monge-Ampère equations and pluripotential theory. In this section we will recall some definition and results of global pluripotential theory in [20, 11, 9, 10, 5].

3.2.1. The complex Monge-Ampère operator and the pluricomplex energy. Let $L \rightarrow$ $X$ be a big line bundle and fix, as above, a smooth Hermitian metric $\|\cdot\|$ on $L$ with curvature form $\theta$. For a smooth curvature form $\theta_{\varphi}\left(:=\theta+d d^{c} \varphi\right)$ one defines the Monge-Ampère measure of the function $\varphi$ (with respect to $\theta$ ) as

$$
M A(\varphi):=\theta_{\varphi}^{n} / V
$$

i.e. the top exterior power of the corresponding curvature form divided by the volume $V$ of the class $[\theta]$ (see below). More generally, according to the classical local pluripotential theory of Bedford-Taylor the expression in formula 3.1 makes locally sense for any bounded $\theta-$ psh function (and the corresponding Monge-Ampère measure does not charge pluripolar subsets, i.e. sets of the form $\{\varphi=-\infty\}$ for a $\theta$-psh function $\varphi$. The corresponding local Monge-Ampère measure is continuous (in the weak topology) with respect to decreasing sequences of bounded $\theta$-psh functions. In general, following [20, for any $\varphi \in P S H(X, \theta)$ we will denote by $M A(\varphi)$ the nonpluripolar Monge-Ampère measure $M A(\varphi)$, which is a globally well-defined measure on $X$ not charging pluripolar subsets and in particular not Zariski closed set. The measure $M A(\varphi)$ is defined by replacing the ordinary wedge products with the so called non-pluripolar products introduced in [20]). In particular, we recall that for $\varphi$ an element in the subspace $P S H(X, \theta)_{\min }$ of functions with minimal singularities the total mass of $\theta_{\varphi}^{n}$ is independent of $\varphi$ and may be taken as the definition of the volume $V$ of the class $[\theta]$. With the normalization in 3.1 this thus means that for 
any $\varphi$ in $P S H(X, \theta)_{\text {min }}$ the measure $M A(\varphi)$ is a probability measure on $X$. On the space $P S H(X, \theta)_{\min }$ there is an energy type functional, denoted by $\mathcal{E}$, which may be defined as a primitive for the one-form defined by $M A(\varphi) / V$, i.e.

$$
d \mathcal{E}_{\mid \varphi}=M A(\varphi),
$$

(in the sense that $d \mathcal{E}(\varphi+t u) / d t=\int_{X} M A(\varphi) u / V$ at $t=0$ ). The functional $\mathcal{E}$ is only defined up to an additive constant which may be fixed by the normalization condition $\mathcal{E}\left(v_{\theta}\right)=0$ for some reference element $v_{\theta}$ in $\operatorname{PSH}(X, \theta)_{\min }$. When $L$ the reference curvature form $\theta$ can be taken to be a Kähler form. Integrating the defining relation 3.2 along a line segment in $\operatorname{PSH}(X, \theta)_{\text {min }}$ reveals that

$$
\mathcal{E}(\varphi)=\frac{1}{(n+1) V} \int_{X} \varphi \sum_{j=0}^{n} \theta_{\varphi}^{j} \wedge \theta_{\varphi}^{n-j}
$$

(but the explicit formula for $\mathcal{E}$ will not really be used in the sequel). Occasionally, we will write $\mathcal{E}_{\theta}$ to indicate the dependence of $\mathcal{E}$ on the fixed normalization. We will also denote by $\mathcal{E}_{\theta}$ the unique upper semi-continuous extension of $\mathcal{E}_{\theta}$ to all of $\operatorname{PSH}(X, \theta)$ and write

$$
\mathcal{E}^{1}(X, \theta):=\left\{\varphi \in \operatorname{PSH}(X, \theta): \mathcal{E}_{\theta}(\varphi)>-\infty\right\},
$$

which is called the space of all functions on $X$ with finite energy. Now following [11] the pluri-complex energy $E_{\theta}(\mu)$ of a probability measure $\mu$ is defined by

$$
E_{\theta}(\mu):=\sup _{\varphi \in P S H(X, \theta)} \mathcal{E}_{\theta}(\varphi)-\langle\varphi, \mu\rangle,
$$

As recalled in the following theorem the sup defining $E_{\theta}$ is in fact attained:

$$
E_{\theta}(\mu):=\mathcal{E}_{\theta}\left(\varphi_{\mu}\right)-\left\langle\varphi_{\mu}, \mu\right\rangle
$$

for a unique function $\varphi_{\mu} \in \mathcal{E}^{1}(X, \theta) / \mathbb{R}$ if $E_{\theta}(\mu)<\infty$ where

$$
M A\left(\varphi_{\mu}\right)=\mu .
$$

Theorem 3.2. 11] The following is equivalent for a probability measure $\mu$ on $X$ :

- $E_{\theta}(\mu)<\infty$

- $\langle\varphi, \mu\rangle<\infty$ for all $\varphi \in \mathcal{E}^{1}(X, \theta)$

- $\mu$ has a potential $\varphi_{\mu} \in \mathcal{E}^{1}(X, \theta)$, i.e. equation 3.5 holds

Moreover, $\varphi_{\mu}$ is uniquely determined $\bmod \mathbb{R}$, i.e. up to an additive constant and can be characterized as the function maximizing the functional whose sup defines $E_{\theta}(\mu)$ (formula 3.3).

Proof. Since the proof fits naturally into the present probabilistic framework we outline its main ingredients. The function $\varphi$ will be obtained by maximizing the following functional on $\mathcal{E}^{1}(X)$ :

$$
\mathcal{G}(\varphi):=\mathcal{E}(\varphi)-\langle\varphi, \mu\rangle
$$

By definition any critical point of $\mathcal{G}$ i.e. function $\varphi$ such that $d \mathcal{G}_{\mid \varphi}=0$ satisfies the previous equation. As usual in the direct method of the calculus of variations there are two main steps:

- The functional $\mathcal{G}$ admits a minimizer $\psi$ on $\mathcal{E}^{1}$

- The minimizer $\psi$ is a critical point. 
The last step would be automatic if $\psi$ were known to be an interior point in the space $\mathcal{E}^{1}$. However, as the latter space is only convex $\psi$ could be in the boundary of $\mathcal{E}^{1}$ viewed as a subset of $P S H(X, \theta)$. To get around this difficulty one observes that, by monotonicity, $\psi$ is also a maximizer of the functional $\tilde{\mathcal{G}}$ obtained by replacing $\mathcal{E}$ with the functional $\mathcal{F}_{\theta}:=\left(\mathcal{E}_{\theta} \circ P_{\theta}\right)$, where $P_{\theta}$ is the projection operator defined below (and which already appeared in the outline in Section 2). The latter functional has the virtue that it is defined on the affine space $\{\psi\}+C^{0}(X)$ and that it is Gateaux differentiable, with a differential given by formula in Theorem 3.6 below. Hence, $d \tilde{\mathcal{G}}_{\mid \psi}=0$, which is equivalent to $d \mathcal{G}_{\mid \psi}=0$, since $P_{\theta} \psi=\psi$.

Coming back to the first step the assumption $E(\mu)<\infty$ means precisely that the sup of $\mathcal{G}$ is finite. To find a minimizer the starting point is the compactness of the space $\operatorname{PSH}(X, \theta)_{0}$ of all sup-normalized functions $\varphi$ (i.e. $\left.\sup _{X} \varphi=0\right)$, using the usual $L^{1}$-topology (which is equivalent to the $L^{p}$-topology for any $p \geq 1$ ). Since $\mathcal{G}$ descends to $\operatorname{PSH}(X, \theta)_{0}$ and the functional $\mathcal{E}$ is lower semi-continuous all that remains is to make sure that the integration pairing $\langle\cdot, \mu\rangle$ is continuous on $\mathcal{E}^{1}(X)$. This follows directly from the compactness of $\operatorname{PSH}(X, \theta)_{0}$ when $\mu$ is a volume form or more generally when $\mu$ has an $L^{p}$-density for some $p>1$. However, for a general $\mu$ of finite energy the proof turns out to be rather subtle. Briefly, one first establishes a general coercivity type inequality of the form

$$
|-\langle\varphi, \mu\rangle| \leq C_{\mu}(-\mathcal{E}(\varphi))^{1 / 2}
$$

It would then be enough to know that that the integration pairing $\langle\cdot, \mu\rangle$ is continuous on any sublevel of the functional $-\mathcal{E}(\varphi)$. Indeed, taking a sup-normalized maximizing sequence $\psi_{j}$ (i.e. tending to the $\sup E(\mu)$ of $\mathcal{G}$ ) the coercivity inequality above ensures that $\psi_{j}$ stays in a fixed sublevel set of $-\mathcal{E}(\varphi)$ and hence, by the lower semi-continuity of $\mathcal{G}$ on such a sublevel set the $L^{1}$-limit in $\operatorname{PSH}(X, \theta)$ is in $\mathcal{E}^{1}$ and maximizes $\mathcal{G}$, as desired. The continuity property of $\langle\cdot, \mu\rangle$ in question is indeed shown in [11. However the proof is rather indirect and tied up with the proof of the existence of a finite energy minimizer.

Example 3.3. In the classical case $n=1$, i.e. $X$ is a Riemann surface and $\theta$ is smooth and strictly positive the space $\mathcal{E}^{1}(X, \theta)$ is the intersection of $\operatorname{PSH}(X, \theta)$ with the vector space of all functions $\varphi$ whose gradient is in $L^{2}(X)$, i.e. $\int d \varphi \wedge$ $d^{c} \varphi<\infty$. Accordingly, using integration by parts, $E_{\theta}(\mu)=\int d \varphi_{\mu} \wedge d^{c} \varphi_{\mu} / 2$. This means that $E_{\theta}(\mu)$ is the classical Dirichlet energy of a charge distribution $\mu$ in the "neutralizing back-ground charge $\theta$ " (compare [3]).

In the case when the class $[\theta]$ is Kähler and $\mu$ is a volume form the existence of a smooth solution to equation 3.5] was first shown by Yau [51] in his celebrated solution of the Calabi conjecture (the uniqueness of such solutions is due to Calabi).

3.2.2. The psh-projection $P_{\theta}$, the functional $\mathcal{F}_{\theta}$ and asymptotics. The "psh-projection" is the operator $P_{\theta}$ from $C^{0}(X)$ to $P S H(X, \theta)_{\min }$ defined as the following envelope:

$$
\left(P_{\theta} u\right)(x):=\sup \{\varphi(x): \varphi \leq u,\}
$$

Using the latter projection operator it will be convenient to take the reference element $v_{\theta}$ in $P S H(X, \theta)_{m i n}$, referred to above, to be defined by

$$
v_{\theta}:=P_{\theta} 0
$$


We may then define the following functional on $C^{0}(X)$ :

$$
\mathcal{F}_{\theta}(u):=\left(\mathcal{E}_{\theta} \circ P_{\theta}\right)(u)
$$

Using the latter functional the pluricomplex energy $E_{\theta}$, defined above, may be realized as a Legendre transform:

Proposition 3.4. The pluricomplex energy $E_{\theta}$ is the Legendre-Fenchel transform of the convex functional $u \mapsto f(u):=-\left(\mathcal{E}_{\theta} \circ P_{\theta}\right)(-u)$, i.e.

$$
E_{\theta}(\mu):=\sup _{u \in C^{0}(X)} \mathcal{E}_{\theta}\left(P_{\theta} u\right)-\langle u, \mu\rangle,
$$

Moreover

$$
E_{\theta}(\mu) \geq 0
$$

with equality precisely for $\mu:=M A\left(v_{\theta}\right)$, where $v_{\theta}:=P_{\theta} 0$.

Proof. This was shown in [20] in the ample case and in [3] in the general big case. Briefly, denoting by $E_{\theta}^{\prime}(\mu)$ the sup appearing in the right hand side of formula 3.7 any $\theta$-psh function of the form $\varphi:=P_{\theta} u$ is a contender for the sup defining $E_{\theta}(\mu)$ and hence, since $P_{\theta}$ is decreasing, $E_{\theta}(\mu) \geq E_{\theta}^{\prime}(\mu)$. To prove the converse first assume that $\mu$ is of the form $\mu=M A\left(P_{\theta} u\right)$ for some $u \in C^{0}(X)$. Then the equality $E_{\theta}(\mu)=E_{\theta}^{\prime}(\mu)$ follows immediately from the general "orthogonality relation" :

$$
\int\left(u-P_{\theta} u\right) M A\left(P_{\theta} u\right)=0
$$

(saying that $M A\left(P_{\theta} u\right)$ is supported on the set $\left\{P_{\theta} u<u\right\}[9]$ ). The case of a general $\mu$ is then proved by approximation [3]. Note that the orthogonality relation also implies that $E_{\theta}\left(M A\left(v_{\theta}\right)\right)=0$.

Remark 3.5. In general, the Legendre-Fenchel transform of a function $f$ on a topological vector spaces $V$ is the convex lsc function $f^{*}$ on the topological dual $V^{*}$ defined by

$$
f^{*}(w):=\sup _{v \in V}\langle v, w\rangle-f(v)
$$

in terms of the canonical pairing between $V$ and $V^{*}$. In the present setting $V=$ $C^{0}(X)$ and $V^{*}=\mathcal{M}(X)$, the space of all signed Borel measures on $X$ (see for example [24]).

As explained in Section 2 a key ingredient in the proof of the large deviation principles described in the introduction of the paper is the following result concerning the existence and differentiability of the transfinite diameter associated to a big Hermitian line bundle (which is equivalent to Theorem A and B in [9]).

Theorem 3.6. 9]. Let $L \rightarrow X$ be a big line bundle equipped with a continuous Hermitian metric $\|\cdot\|$ on $L$ with curvature current $\theta$. Then

- If $\operatorname{det} S^{(k)}$ denotes the element in the determinant line of $H^{0}(X, k L)$ induced by a basis in $H^{0}(X, k L)$ which is orthonormal with respect to the $L^{2}$-norm determined by $\|\cdot\|$ and a volume form $d V$ on $X$ (or more generally a measure $\mu_{0}$ which has the Bernstein-Markov property) then

$$
\lim _{k \rightarrow \infty}-\frac{1}{k N_{k}} \sup _{X^{N}}\left(\log \left\|\operatorname{det} S^{(k)}\right\|^{2}\left(x_{1}, \ldots, x_{N}\right)+k u\left(x_{1}\right)+\cdots+k u\left(x_{N}\right)\right)=\mathcal{F}_{\theta}(u),
$$


- The functional $\mathcal{F}_{\theta}$ is Gateaux differentiable on $C^{0}(X)$ with differential

$$
\left(d \mathcal{F}_{\theta}\right)_{\mid u}=M A\left(P_{\theta} u\right) .
$$

Proof. As a courtesy to the reader we outline the proof of the theorem, starting with the case when $L$ is ample (compare the end of Section 4 in [9]). Fix a volume form $d V$ on $X$. Given a continuous metric $\phi$ on $L$ we set

$$
\mathcal{F}_{k, L^{p}}[\phi]:=-\frac{1}{k N_{k}} \log \left\|\operatorname{det} S^{(k)}\right\|_{L^{p}\left(X^{\left.N_{k}, k \phi, d V\right)}\right.}^{2}
$$

defined in terms of $L^{p}$-norm on $H^{0}\left(X^{N},(k L)^{N_{k}}\right)$ induced by $(\phi, d V)$, for $p \in[1, \infty]$ (which is defined to be the ordinary sup-norm for $p=\infty$ and thus independent of $d V)$. For $p=\infty$, which is the case appearing in the statement of the theorem, it follows immediately from the definitions that

$$
\mathcal{F}_{k, L^{\infty}}[\phi]=\mathcal{F}_{k, L^{\infty}}[P \phi]
$$

and that $\mathcal{F}_{k, L^{\infty}}$ is equicontinuous with respect to the sup-norm on the space of continuous metrics. Accordingly, writing the positively curved metric $P \phi$ as the uniform limit of smooth and positively curved metrics $\psi_{j}$ (using Demailly's approximation theorem on an ample line) it is enough to prove the convergence of $\mathcal{F}_{k, L \infty}[\psi]$ when $\psi$ is a smooth metric with strictly positive curvature. To this end one uses that

$$
\mathcal{F}_{k, L \infty}[\psi]=\mathcal{F}_{k, L^{2}}[\psi]+o(1),
$$

where the error term $o(1)$ (tending to zero) only depends on the modulus of continuity of $\psi$. Indeed, this follows directly from applying the standard submean property of holomorphic functions on small coordinate balls on $X$, for each factor of $X^{N_{k}}$. Now, a direct calculation reveals that the differential of $\mathcal{F}_{k, L^{2}}$ at any metric $\psi$ is given by

$$
d\left(\mathcal{F}_{k, L^{2}}\right)_{\mid \psi}=\frac{1}{N_{k}} \rho_{k \psi} d V
$$

where the function $\rho_{k \psi}$ is the restriction to the diagonal of the point-wise norm of the Bergman kernel of the Hilbert space $\left(H^{0}(X, k L),\|\cdot\|_{L^{2}(X, d V)}\right)$. The asymptotics of $\rho_{k \psi}$, when $\psi$ is a smooth metric with strictly positive curvature, are well-known and in particular give that

$$
\text { (i) } \lim _{k \rightarrow \infty} \frac{1}{N_{k}} \rho_{k \psi} d V=\frac{1}{V}\left(d d^{c} \psi\right)^{n}(i i) \frac{1}{N_{k}} \rho_{k \psi} \leq C
$$

in the weak topology. Using the defining property 3.2 of the functional $\mathcal{E}$ (and integrating along a line segment in the space of all positively curved metrics) this gives $\mathcal{F}_{k, L^{\infty}}[\psi]=\mathcal{E}(\psi)+o(1)$, which proves the first point in the theorem, thanks to 3.10 and 3.11 .

As for the differentiability in the second point it is proved in [9] in the general setting of $\theta$-psh functions, not necessarily associated to a line bundle (i.e. for a a general big class $\left.[\theta] \in H^{1,1}(X . \mathbb{R})\right)$, using the orthogonality relation 3.8. However in the present line bundle setting an alternative proof using the general Bergman kernel asymptotics in 2] can be given. Indeed, by [2,

$$
d\left(\mathcal{F}_{k, L^{2}}\right)_{\mid \phi}=\frac{1}{V}\left(d d^{c} P \phi\right)^{n}+o(1)
$$


for any smooth metric $\phi$ on $L$ (thus generalizing 3.12). Using 3.10 and 3.11 again this implies the desired differentiability result. In the general case of a line bundle $L$ which is merely big one cannot reduce the problem to the case of smooth and positively curved metrics (since such metrics will not, in general, exist). But the point is that, as shown in [2, the asymptotics 3.13 are always valid for any big line bundle, which is enough to conclude (also using the general differentiability result in $[9]$ ).

The next lemma provides the regularization property 2.6 in the present setting:

Lemma 3.7. Let $\mu$ be a probability measure such that $E_{\theta}(\mu)$. Then there exists a sequence $\mu_{j}$ of probability measures of the form $\mu_{j}=M A\left(P_{\theta} u_{j}\right)$, for $u_{j} \in C^{0}(X)$, such that

$$
\lim _{j \rightarrow \infty} \mu_{j}=\mu, \lim _{j \rightarrow \infty} E\left(\mu_{j}\right)=\mu
$$

where the first convergence holds in the weak topology.

Proof. If $E(\mu)<\infty$, then by Theorem 3.2 we can write $\mu=M A(\varphi)$ for a function $\varphi$ with finite energy. Since the function $\varphi$ is usc it is a decreasing limit of continuous functions $u_{j}$ on $X$. It then follows, by monotonicity, that the projections $P u_{j}$ also decrease to $\varphi$ and hence, by the continuity of mixed Monge-Ampère expression under monotone limits [20] it follows that $\mu_{j}:=M A\left(P u_{j}\right) \rightarrow \mu$ and $E\left(\mu_{j}\right) \rightarrow E(\mu)$, as desired.

The previous lemma can also be obtained form general properties of Legendre transforms (see [8, Lemma 3.1]).

\section{THE LDP FOR $\beta$-DEFORMED DETERMINANTAL POINT PROCESSES}

Given a compact topological space $X$ we will denote by $\mathcal{M}(X)$ the space of all signed (Borel) measures on $X$ and by the $\mathcal{M}_{1}(X)$ the subspace of all probability measures, i.e. $\mu \geq 0$ and $\int_{X} \mu=1$. We endow $\mathcal{M}(X)$ with the weak topology, i.e. $\mu_{j}$ is said to converge to $\mu$ weakly in $\mathcal{M}(X)$ if

$$
\left\langle u, \mu_{j}\right\rangle \rightarrow\langle u, \mu\rangle
$$

for any continuous function $u$ on $X$, i.e. for any $u \in C^{0}(X)$, where $\langle u, \mu\rangle$ denotes the standard integration pairing between $C^{0}(X)$ and $\mathcal{M}(X)$.

4.1. Probabilistic preliminaries. A probability space is a space $\Omega$ equipped with a probability measure $\mu$. The space $\Omega$ is called the sample space and a measurable subset $\mathcal{B} \subset \Omega$ is called an event with

$$
\operatorname{Prob} \mathcal{B}:=\mu(\mathcal{B})
$$

interpreted as the probability of observing the event $\mathcal{B}$ when sampling from $(\mathcal{X}, \Omega)$. A measurable function $Y: \Omega \rightarrow \mathcal{Y}$ on a probability space $(\Omega, \mu)$ is called a random element with values in $Y$ and its law $\Gamma$ is the probability measure on $\mathcal{Y}$ defined by the push-forward measure

$$
\Gamma:=Y_{*} \mu
$$

(the law of $Y$ is often also called the distribution of $Y$ ). A sequence of random elements $Y_{N}: \Omega_{N} \rightarrow \mathcal{Y}$ taking values in the same topological space $\mathcal{Y}$ are said 
to convergence in law towards a deterministic element $y$ in $Y$ if the corresponding laws $\Gamma_{N}$ on $\mathcal{Y}$ converge to a Dirac mass at $y$ :

$$
\lim _{N \rightarrow \infty} \Gamma_{N}=\delta_{y}
$$

in the weak topology. If $\mathcal{Y}$ is a separable metric space with metric $d$ then $Y_{N}$ converge in law towards the deterministic element $y$ iff $Y_{N}$ converge in probability towards $y$, i.e. for any fixed $\epsilon>0$ i.e.

$$
\lim _{N \rightarrow \infty} \operatorname{Prob}\left\{d\left(Y_{N}, y\right)>\epsilon\right\}=0 .
$$

4.1.1. Random point processes. A random point process with $N$ particles on a space $X$ is, by definition, a probability measure $\mu^{(N)}$ on the $N$-fold product $X^{N}$ (the $N$-particle space) which is symmetric, i.e. invariant under action of the group $S_{N}$ by permutations of the factors of $X^{N}$. Its j-point correlation measure $\mu_{j}^{(N)}$ is the probability measure on $X^{j}$ defined as the push forward of $\mu^{(N)}$ to $X$ under the map $X^{N} \rightarrow X^{j}$ given by projection onto the first $j$ factors (or any $j$ factors, by symmetry):

$$
\mu_{j}^{(N)}:=\int_{X^{N-j}} \mu^{(N)}
$$

The empirical measure of a given random point process is the following random measure

$$
\delta_{N}: X^{N} \rightarrow \mathcal{M}_{1}(X), \quad\left(x_{1}, \ldots, x_{N}\right) \mapsto \delta_{N}\left(x_{1}, \ldots, x_{N}\right):=\frac{1}{N} \sum_{i=1}^{N} \delta_{x_{i}}
$$

on $\left(X^{N}, \mu^{(N)}\right)$. The law of $\delta_{N}$ thus defines a probability measure on the space $\mathcal{M}_{1}(X)$ that we shall denote by $\Gamma_{N}$.

Lemma 4.1. Consider a sequence of random point processes with $N$ particles on $X$. Then the corresponding random measures $\delta_{N}$ converge in law towards a deterministic measure $\mu$ iff

$$
\lim _{N \rightarrow \infty} \mu_{j}^{(N)}=\mu^{\otimes j}
$$

weakly on $X^{j}$, for any fixed positive integer $j$.

Proof. For completeness we give the simple proof of the convergence of the $j$-point correlation measures under the assumption that $\delta_{N}$ converge in law towards a deterministic measure $\mu$ (which is the direction we will be interested in). When $j=1$ the convergence 4.2 means that, for any given $u \in C^{0}(X)$ the following holds:

$$
\lim _{N \rightarrow \infty} \int_{X^{N}} \frac{1}{N}\left(u\left(x_{1}\right)+\ldots+u\left(x_{N}\right)\right) \mu^{(N)}=\int_{X} u \mu .
$$

Denoting by $\mathcal{U}$ the continuous function on $\mathcal{M}(X)$ defined by $\mathcal{U}(\mu):=\int_{X} u \mu$, for a given $u \in C^{0}(X)$, the integral in the left hand side above may be written as

$$
\int_{X^{N}}\left(\delta_{N}^{*} \mathcal{U}\right) \mu^{(N)}=\int_{\mathcal{M}_{1}(X)} \mathcal{U} \Gamma_{N}, \Gamma_{N}:=\left(\delta_{N *} \mu^{(N)}\right),
$$

which, by assumption, converges to $U\left(\delta_{\mu}\right):=\int_{X} u \mu$, as $N \rightarrow \infty$, as desired. The case of $j>1$ is proved in a similar way by replacing the linear function $\mathcal{U}$ with a "multinomial" $\mathcal{U}_{j}(\mu):=\int_{X} u_{1}\left(x_{1}\right) \mu \cdots \int_{X} u_{j}\left(x_{1}\right) \mu$ determined by $j$ given elements $u_{j}$ in $C^{0}(X)$. 
4.1.2. The notion of a Large Deviation Principle (LDP). The notion of a Large Deviation Principle (LDP), introduced by Varadhan, allows one to give a notion of exponential convergence, which can be seen as an infinite dimensional version of the Laplace principle [24]. Let us first recall the general definition of a Large Deviation Principle (LDP) for a general sequence of measures.

Definition 4.2. Let $\mathcal{P}$ be a Polish space, i.e. a complete separable metric space.

(i) A function $I: \mathcal{P} \rightarrow]-\infty, \infty]$ is a rate function if it is lower semi-continuous. It is a good rate function if it is also proper.

(ii) A sequence $\Gamma_{k}$ of measures on $\mathcal{P}$ satisfies a large deviation principle with speed $r_{k}$ and rate function $I$ if

for any closed subset $\mathcal{F}$ of $\mathcal{P}$ and

$$
\limsup _{k \rightarrow \infty} \frac{1}{r_{k}} \log \Gamma_{k}(\mathcal{F}) \leq-\inf _{\mu \in \mathcal{F}} I
$$

$$
\liminf _{k \rightarrow \infty} \frac{1}{r_{k}} \log \Gamma_{k}(\mathcal{G}) \geq-\inf _{\mu \in G} I(\mu)
$$

for any open subset $\mathcal{G}$ of $\mathcal{P}$.

The simplest instance of an LDP appears when $\mathcal{P}=\mathbb{R}^{n}$ and $\Gamma_{k}(y)$ is a probability measure of the form $\Gamma_{k}(y)=e^{-r_{k} I(y)} d y / Z_{k}$ for an appropriate lower semicontinuous function $I$.

Example 4.3. LLet $Y_{1}, \ldots, Y_{N}$ be $N$ independent normal standard random variables, i.e. Gaussian random variables with zero mean and unit variance. Then the law of the corresponding sample mean $\bar{Y}_{N}=\left(Y_{1}+\ldots Y_{N}\right) / N$ satisfies a LDP on $\mathbb{R}$ with rate functional $I(y)=|y|^{2} / 2$ and speed $N$. Indeed, by definition each $Y_{i}$ is the coordinate variable on the probability space $(\mathbb{R}, \gamma)$, where $\gamma$ is the standard Gaussian probability measure, i.e. $\gamma=(2 \pi)^{-1 / 2} e^{-|y|^{2}} d y$ and an explicit calculation gives the exact formula

$$
\Gamma_{N}:=\left(Y^{(N)}\right)_{*} \gamma^{\otimes N}=(2 \pi)^{-1 / 2} e^{-N|y|^{2} / 2} d y,
$$

which implies the LDP in question. More generally, by Cramér's theorem [24, replacing $\gamma$ with any probability measure $\nu$ (with finite exponential moments) gives an LDP with speed $N$ and a rate function $I(y)$ which, by inverting the Laplace transform of $\nu$, may be represented as follows, in terms of the one-dimensional Legendre-Fenchel transform (see Remark 3.5):

$$
I(y):=f^{*}(y), \quad f(x):=\log \int_{\mathbb{R}} e^{\langle x, y\rangle} \nu(y),
$$

In particular, by convexity, $I(y)$ vanishes precisely on $d f / d x_{\mid x=0}=\int y \nu$. This implies the weak (and also strong) law of large numbers saying that $\bar{Y}_{N}$ converges in probability (and even almost surely) to the deterministic value $\int y \nu$; indeed, the sample mean is even exponentially concentrated around its expectation.

Let us also mention the following classical infinite dimensional generalization of the previous LDP due to Mogulskii (see [24, Chapter 5]):

Example 4.4. Set $\bar{Y}_{N}(t):=\bar{Y}_{[t N]}$ for $t \in[0,1]$ where $[c]$ denotes the integer part of $c$. If $Y_{i}$ are standard independent normal random variables, then the law $\Gamma_{N}$ of $\bar{Y}_{N}(t)$, viewed as random element with values in the space $C^{0}[0,1]_{0}$ of all continuous 
functions on $[0,1]$ such that $u(0)=0$, satisfies an LDP with speed $N$ and rate functional defined by

$$
I(u):=\int_{0}^{1}\left|\frac{d u}{d t}\right|^{2} d t
$$

if the function $u \in C^{0}[0,1]_{0}$ has a distributional derivative in $L^{2}[0,1]$ and otherwise $I(u)=\infty$. Interpreting the parameter $t$ as time the random function $\bar{Y}_{N}(t)$ can be viewed as a sample path for a random walk on $\mathbb{R}$ starting at the origin.

We will be mainly interested in the case when $\Gamma_{k}$ is a probability measure (which implies that $I \geq 0$ with infimum equal to 0 ). Then it will be convenient to use the following alternative formulation of a LDP (see Theorems 4.1.11 and 4.1.18 in [24]):

Proposition 4.5. Let $\mathcal{P}$ be a compact metric space and denote by $B_{\epsilon}(\nu)$ the ball of radius $\epsilon$ centered at $\nu \in \mathcal{P}$. Then a sequence $\Gamma_{N}$ of probability measures on $\mathcal{P}$ satisfies a LDP with speed $r_{N}$ and a rate functional I iff

$$
\lim _{\epsilon \rightarrow 0} \liminf _{N \rightarrow \infty} \frac{1}{r_{N}} \log \Gamma_{N}\left(B_{\epsilon}(\nu)\right)=-I(\nu)=\lim _{\epsilon \rightarrow 0} \limsup _{N \rightarrow \infty} \frac{1}{r_{N}} \log \Gamma_{N}\left(B_{\epsilon}(\nu)\right)
$$

In particular, if the rate functional $I$ has a unique minimizer $\mu_{\min }$, then $\Gamma_{N} \rightarrow$ $\delta_{\mu_{\text {min }}}$, weakly, as $N \rightarrow \infty$.

In the present setting $\Gamma_{N}$ will arise as the law of the empirical measures $\delta_{N}$ and the rate functional $I$ will be shown to have a unique minimizer, which will thus imply that $\delta_{N}$ converges in law towards the deterministic measure $\mu_{\text {min }}$.

We will have great use for the following classical result of Sanov, which is the standard example of an LDP for point processes.

Proposition 4.6. (Sanov) Let $X$ be a topological space and $\mu_{0}$ a finite measure on $X$. Then the laws $\Gamma_{N}$ of the empirical measures $\delta_{N}$ defined with respect to the product measure $\mu_{0}^{\otimes N}$ on $X^{N}$ satisfy an LDP with speed $N$ and rate functional the relative entropy $D_{\mu_{0}}$.

Proof. We recall that, as shown in [24], the proof can be obtained from the infinite dimensional generalization of Cramér's theorem in example 4.3. Indeed, assuming for simplicity that $X$ is compact (which will be the case in the present setting) the topological dual of the topological vector space $\mathcal{M}(X)$, consisting of signed Borel measures, may be identified with $C^{0}(X)$ and the rate functional $I$ on $\mathcal{M}(X)$ may be written as the Legendre-Fenchel transform $f^{*}(\mu)$, where $f(u)=\log \int e^{\left\langle u, \delta_{x}\right\rangle} \mu_{0}=$ $\log \int e^{u(x)} \mu_{0}$. A direct computation then reveals that $f^{*}$ is the relative entropy.

We recall that the relative entropy $D_{\mu_{0}}$ (also called the Kullback-Leibler divergence or the information divergence in probability and information theory) is the functional on $\mathcal{M}_{1}(X)$ defined by

$$
D_{\mu_{0}}(\mu):=\int_{X} \log \frac{\mu}{\mu_{0}} \mu,
$$

when $\mu$ has a density $\frac{\mu}{\mu_{0}}$ with respect to $\mu_{0}$ and otherwise $D_{\mu_{0}}(\mu):=\infty$. When $\mu_{0}$ is a probability measure, $D_{\mu_{0}}(\mu) \geq 0$ and $D_{\mu_{0}}(\mu)=0$ iff $\mu=\mu_{0}$ (by Jensen's inequality). 
4.1.3. Gibbs measures. Let $X$ be a compact topological space endowed with a measure $\mu_{0}$ and $H^{(N)}\left(x_{1}, \ldots, x_{N}\right)$ a symmetric function on $X^{N}$. As recalled in Section 2.4. for any given positive number $\beta$ the corresponding Gibbs measure at inverse temperature $\beta$

$$
\mu_{\beta}^{(N)}:=e^{-\beta H^{(N)}} \mu_{0}^{\otimes N} / Z_{N, \beta}, Z_{N . \beta}:=\int_{X^{N}} e^{-\beta_{N} H^{(N)}} \mu_{0}^{\otimes N},
$$

defines a random point process on $X$ with $N$ particles, assuming that the normalizing constant $Z_{N, \beta}$ (called the partition function) is finite. We note the following lemma which allows one to extend Prop 4.5 to the non-normalized measures $\left(\delta_{N}\right)_{*} e^{-\beta H^{(N)}} \mu_{0}^{\otimes N}$ (see [8] for the simple proof).

Lemma 4.7. Assume that $\left|\log Z_{N, \beta}\right| \leq C N$. Then the measures $\left(\delta_{N}\right)_{*} e^{-\beta H^{(N)}} \mu_{0}^{\otimes N}$ satisfy the asymptotics 4.3 for any $\nu \in \mathcal{M}_{1}(X)$ with rate functional $\tilde{I}(\mu)$ and speed $N$ iff the probability measures $\left(\delta_{N}\right)_{*} \mu_{\beta}^{(N)}$ on $\mathcal{M}_{1}(X)$ satisfy an LDP at speed $N$ with rate functional $I:=\tilde{I}-C_{\beta}$, where $C_{\beta}:=\inf _{\mu \in \mathcal{M}(X)} I(\mu)$.

4.2. Large deviations for $\beta$-deformation of Vandermonde type determinants attached to a big line bundle $L$. As above we let $L \rightarrow X$ be a given big line bundle over a compact complex manifold, $\|\cdot\|$ is a smooth Hermitian metric on $L$ (whose curvature current will be denoted by $\theta$ ). We also fix a finite measure $\mu_{0}$ on $X$ a positive number $\beta$. To this data we may associate the following sequence of probability measures on $X^{N_{k}}$ :

$$
\mu^{\left(N_{k}, \beta\right)}:=\frac{\left\|\left(\operatorname{det} S^{(k)}\right)\left(x_{1}, x_{2}, \ldots x_{N_{k}}\right)\right\|^{2 \beta / k} \mu_{0}^{\otimes N_{k}}}{Z_{N_{k}, \beta}}
$$

where we recall that $N_{k}$ is the dimension of $H^{0}(X, k L)$ and $\operatorname{det} S^{(k)}$ is a generator of the corresponding determinant line $\Lambda^{N_{k}} H^{0}(X, k L)$ viewed as a one-dimensional subspace of $H^{0}\left(X^{N_{k}},(k L)^{\otimes N_{k}}\right)$ (the totally anti-symmetric part). As usual, $Z_{N_{k}, \beta}$ is the normalizing constant (partition function):

$$
Z_{N_{k}, \beta}:=\int_{X^{N_{k}}}\left\|\operatorname{det} S^{(k)}\right\|^{2 \beta / k} \mu_{0}^{\otimes N_{k}}
$$

By homogeneity $\mu^{\left(N_{k}, \beta\right)}$ is independent of the choice of generator $\operatorname{det} S^{(k)}$. It will be convenient to take $\operatorname{det} S^{(k)}$ to be the generator determined by a basis in $H^{0}(X, k L)$ which is orthonormal with respect to the $L^{2}$-product determined by $(\|\cdot\|, d V)$ for any fixed volume form $d V$ on $X$.

Remark 4.8. More generally, one can let $\beta$ depend on $N$ (i.e. on $k$ ) in the definition of $\mu^{\left(N_{k}, \beta\right)}$. When $\beta_{N_{k}}=k$ the corresponding probability measure $\mu^{\left(N_{k}, \beta_{k}\right)}$ defines a determinantal point processes, i.e. its density can be written as

$$
\left\|\operatorname{det}_{i, j \leq N}\left(K^{(k)}\left(x_{i}, x_{j}\right)\right)\right\| / N_{k} !,
$$

where $K^{(k)}(x, y)$ denotes the kernel of the orthogonal projection onto the space $H^{0}(X, k L)$ viewed as a subspace of the space $C^{\infty}(X, k L)$ of all smooth sections equipped with the $L^{2}$-norm determined by $\left(\|\cdot\|, \mu_{0}\right)$. There is an extensive literature concerning general properties of determinantal point process ( defined with respect to general Hilbert spaces of functions in $L^{2}\left(\mu_{0}\right)$ for a given measure $\mu_{0}$ on a space $X$ ) - for example, all the $j$-point correlation measures can be expressed as 
determinants involving the kernel $K(x, y)$. The LDP for determinantal point processes associated to a line bundle $L \rightarrow X$ as above was established in 3] for very general measures $\mu_{0}$ and can be viewed as a zero-temperature limit since $\beta_{N_{k}} \rightarrow \infty$ (compare Section 2.4). However, the present setting, which more generally applies when $\beta_{k}$ has the asymptotics $\beta_{k}=\beta+o(1)$ for a positive number $\beta$, appears to be substantially more involved from an analytic point of view and we will only be able to establish the LDP in question for sufficiently regular measures $\mu_{0}$. The case $\beta=1$ is singled out by the fact that it allows the construction of canonical point processes independent of any geometric back-ground data, when $L$ is the canonical line bundle on a variety of positive Kodaira dimension (as explained in Section 5.1).

The probability measure above is thus the Gibbs measure defined by the following Hamiltonian on $X^{N_{k}}$ :

$$
E^{\left(N_{k}\right)}\left(x_{1}, x_{2}, \ldots x_{N_{k}}\right):=-\frac{1}{k} \log \left\|\left(\operatorname{det} S^{(k)}\right)\left(x_{1}, x_{2}, \ldots x_{N_{k}}\right)\right\|^{2}
$$

Since the metric $\|\cdot\|$ is determined up to a multiplicative constant by its curvature current $\theta$, the probability measures above thus only depends on the metric through its curvature and is hence determined by the triple $\left(\mu_{0}, \theta, \beta\right)$. To the latter triple we may also attach the following free energy type functional on the space $\mathcal{M}_{1}(X)$ of all probability measures on $X$ :

$$
F_{\beta}=E_{\theta}+\frac{1}{\beta} D_{\mu_{0}}
$$

Note that the energy functional $E_{\theta}$ is minimized on the measure $M A\left(v_{\theta}\right)$ (see Prop 3.4), while the entropy functional $D_{\mu_{0}}$ is minimized on $\mu_{0}$. By [5, 12] the minimizers of $F_{\beta}$ may be identified with solutions to a complex Monge-Ampère equation (the existence of smooth solutions for an ample line bundle was first shown in the seminal works of Aubin [1] and Yau [51]):

Proposition 4.9. Let $[\theta]$ be a big class (for example, $[\theta]=c_{1}(L)$ for $L$ a big line bundle) and consider the free energy functional $F_{\beta}$ attached to the triple $\left(\mu_{0}, \theta, \beta\right)$ (for $\beta>0)$, where $\mu_{0}$ has finite energy. Then any minimizer $\mu_{\beta}$ of $F_{\beta}$ on $\mathcal{M}_{1}(X)$ can be written as $\mu_{\beta}=M A\left(u_{\beta}\right)$ where $u_{\beta}$ is the unique finite energy solution of the equation

$$
M A\left(u_{\beta}\right)=e^{\beta u_{\beta}} \mu_{0}
$$

Proof. The case of a Kähler class was proved in [5] and the proof generalizes word for word to the case of a big case. For completeness we outline the proof. First of all, by the strict convexity of the relative entropy and the convexity of $E$ the functional $F_{\beta}$ is strictly convex and in particular if a minimizer $\mu_{m i n}$ exists, then it it unique. In fact, by the lower semi-continuity of $F_{\beta}$ there always exists a minimizer and as we will next show it can be obtained from the equation in the proposition. To see this we recall that, as shown in [5], given any $\mu \in \mathcal{P}(X)$ such that $E(\mu)<\infty$, the function $-\varphi_{\mu}$ is a subgradient for the convex functional $E(\mu)$ at $\mu$ in the sense that

$$
E(\nu) \geq E(\mu)+\int_{X}\left(-\varphi_{\mu}\right)(\nu-\mu)
$$

for any $\nu \in \mathcal{P}(X)$ (the proof in the case of a big class is the same, as it relies on [11] which holds in the general setting of a big class). Moreover, it is a classical 
fact that if $D_{\mu_{0}}(\mu)<\infty$ then $\log \left(\mu / \mu_{0}\right)$ is a subgradient (and even a gradient) for $D_{\mu_{0}}(\mu)$. Hence, if $\mu$ satisfies the equation

$$
\frac{1}{\beta} \log \left(\mu / \mu_{0}\right)-\varphi_{\mu}=0,
$$

then $F_{\beta}(\nu) \geq F_{\beta}(\mu)$ for any $\nu \in \mathcal{P}(X)$, i.e. $\mu$ minimizes $F_{\beta}(\mu)$. Now, if $\varphi \in \mathcal{E}^{1}(X, \theta)$ solves the equation 4.9 then $\mu:=M A\left(\varphi_{\mu}\right)$ solves the equation 4.11 so all that remains is to find a solution to the equation 4.9 in $\mathcal{E}^{1}(X, \theta)$. But the existence of such a solution follows from the results in [1] (generalizing Theorem 3.2 which corresponds to $\beta=0$ ). In a nutshell, the function $\varphi$ is obtained by maximizing the following lsc functional on $\mathcal{E}^{1}(X, \theta)$ :

$$
\mathcal{E}(\psi)-\frac{1}{\beta} \log \int e^{\beta \psi} \mu_{0} .
$$

For the complex geometric applications it will be adequate to consider measures of the following form:

$$
\mu_{0}=e^{\psi_{+}-\psi_{-}} d V
$$

where $d V$ is a volume form on $X$ and $\psi_{ \pm}$are quasi-psh functions.

Remark 4.10. When $L$ is ample and $\mu_{0}$ is a singular volume, which is singular and degenerate along a klt divisor $\Delta$, in $X$ it is well-known that the solution is smooth away from the support of $\Delta$ (more generally, the regularity holds on the intersection with the ample locus of $L$ when $L$ is assumed merely nef [20]). However, for the purposes of the present paper such regularity properties will not play any role.

Theorem 4.11. Let $L \rightarrow X$ be a big line equipped with a continuous Hermitian metric $\|\cdot\|$ and $\mu_{0}$ a finite measure on $X$ of the form 4.12. Fix a positive number $\beta$. Then the empirical measures of the corresponding random point processes on $X$ converge in probability towards the measure $\mu_{\beta}$, where $\mu_{\beta}=M A\left(u_{\beta}\right)$ for the unique solution $u_{\beta}$ of the complex Monge-Ampère equation 4.9. More precisely:

- The non-normalized measures $\left(\delta_{N}\right)_{*}\left(\left\|\operatorname{det} S^{(k)}\right\|^{2 \beta / k} \mu_{0}^{\otimes N_{k}}\right)$, where $\operatorname{det} S^{(k)}$ an element in the determinant line of $H^{0}(X, k L)$ induced by a basis in $H^{0}(X, k L)$ which is orthonormal with respect to the $L^{2}$-norm determined by $\left(\|\cdot\|, \mu_{0}\right)$ satisfy a LDP on $\mathcal{M}_{1}(X)$ with rate functional $F_{\beta}$ and the corresponding partition functions satisfy

$$
-\lim _{N_{k} \rightarrow \infty} \frac{1}{N_{k}} \log Z_{N_{k}, \beta}=\inf _{\mathcal{M}_{1}(X)} F_{\beta}=: C_{\beta}
$$

- As a consequence, the laws of the empirical measures of the random point processes defined by formula 4.5 satisfy a large deviation principle (LDP) with speed $\beta N$ and rate functional $F_{\beta}(\mu)-C_{\beta}$.

Proof. When $\mu_{0}$ is a volume form the proof of the first point was outlined in Section 2 and the full proof appears in the companion paper 8 . Here we just point out that the proof also applies to more general $\mu_{0}$ of the form 4.12. First of all, the proof of the upper bound was given in Section 2 and did not use any properties of the measure $\mu_{0}$ at all. As for the lower bound we first observe that, upon replacing $\beta H^{(N)}$ with $\beta H^{(N)}\left(x_{1}, \ldots x_{N}\right)-\psi_{+}\left(x_{1}\right)-\ldots .-\psi_{+}\left(x_{N}\right)$, we may as well 
assume that $\mu_{0}=e^{-\psi_{-}} d V$. Now, since $\psi_{-}$is assumed usc there is a sequence $v_{j} \in$ $C^{0}(X)$ decreasing to $\psi_{-}$and we set $\mu_{0, j}:=e^{-v_{j}} d V$. We thus have $e^{-\beta H^{(N)}} \mu_{0}^{\otimes N} \geq$ $e^{-\beta H^{(N)}} \mu_{0, j}^{\otimes N}$, so that we can apply the LDP with respect to $\mu_{0, j}$ for a fixed $j$ to get that the liminf in the LDP with respect to $\mu_{0}$ is bounded from below by $\mathcal{F}^{*}(\mu)+D_{\mu_{0, j}}(\mu) / \beta$. Finally, the proof is concluded by letting $j \rightarrow \infty$ and using the monotone convergence theorem of integration theory. The LDP in the second point then follows from Lemma 4.7 .

Remark 4.12. Note that when $\beta=1$ the probability measure $\mu^{(N, \beta)}$ on $X^{N}$ is invariant under the transformation $\left(\|\cdot\|^{2}, \mu_{0}\right) \mapsto\left(\left(\|\cdot\|^{2} e^{-v}, e^{v} \mu_{0}\right)\right.$ of the defining data. Hence, by the previous theorem the corresponding free energy functional $F_{\beta}$ is invariant under $\left(\theta, \mu_{0}\right) \mapsto\left(\theta+d d v, e^{v} \mu_{0}\right)$, up to an additive constant. In fact, this is easy to see directly, since it follows from the definitions that $D_{e^{v} \mu_{0}}(\mu)=$ $D_{\mu_{0}}(\mu)-\int v \mu$ and $E_{\theta+d d^{c} v}(\mu)=E_{\theta}(\mu)+\int v \mu-C_{v}$ (where the constant $C_{v}$ ensures that the infimum of $E_{\theta+d d^{c} v}$ vanishes.

4.3. Identification of $F_{\beta}$ with Mabuchi's K-energy functional. Now assume that $L$ is ample and fix data $(\|\cdot\|, d V, \beta)$ where $d V$ denotes a fixed volume form. Since $L$ is ample we may assume that the curvature form of $\|\cdot\|$ is a Kähler form that we denote by $\omega_{0}$. The probability measures 4.5 only depends on the data through the following two form

$$
\eta:=\beta \omega_{0}+\operatorname{Ric} \mu_{0},
$$

where Ric $\mu_{0}$ denotes the curvature form of the metric on $-K_{X}$ induced by the volume form $\mu_{0}$ (compare Remark 4.12). It is a basic fact that the Monge-Ampère equation 4.9 is equivalent to the twisted Kähler-Einstein equation

$$
\operatorname{Ric} \omega=-\beta \omega+\eta,
$$

(see [8, Lemma 5.1]).

We denote by $\mathcal{H}\left(X, \omega_{0}\right)$ the interior of $P S H\left(X, \omega_{0}\right) \cap C^{\infty}(X)$. The map $\varphi \mapsto \omega_{\varphi}$ identifies $\mathcal{H}\left(X, \omega_{0}\right) / \mathbb{R}$ with the space of Kähler metrics in $c_{1}(L)$. By the Calabi-Yau theorem [51 any normalized volume form $\mu$ on $X$ is the volume form of a unique Kähler form $\omega \in c_{1}(L)$, i.e. $\mu=\omega^{n}$ for a Kähler form $\omega \in c_{1}(L)$. As explained in [5, under the corresponding Calabi-Yau isomorphism $\mu \mapsto \omega$, the functional $F_{\beta}$ may be identified with the twisted version of Mabuchi's K-energy $\kappa$ of $\omega$, i.e.

$$
F_{\beta}\left(\omega^{n}\right)=\kappa(\omega),
$$

We recall that the functional $\kappa$ was originally defined in [37, for a general polarized manifold $(X, L)$ (when $\eta=0$ ) by the implicit property

$$
d\left(\kappa\left(\omega_{\varphi}\right)_{\mid \varphi}=-\left(R_{\omega_{\varphi}}-C\right) \omega_{\varphi}^{n}\right.
$$

for $\varphi \in \mathcal{H}\left(X, \omega_{0}\right)$, where $R_{\omega_{\varphi}}$ is the (normalized) scalar curvature of the Kähler metric $\omega_{\varphi}$ and $C$ is a constant only depending on the cohomology of $(X, L)$. This formula thus defines $\kappa$ up to a normalizing constant. When $L=K_{X}$ the formula 4.15 then follows directly from the Chen-Tian formula for $\kappa$. For the convenience of the reader we note that a direct proof of formula 4.15 can be given using Legendre transforms as follows (see Proposition 3.4). When $\varphi \in \mathcal{H}\left(X, \omega_{0}\right)$ setting $\mu=\omega_{\varphi}^{n}$ gives

$$
E_{\omega_{0}}(\mu)=f^{*}\left(d f_{\mid(-\varphi)}\right)
$$


and hence, by basic properties of Legendre transform, $d\left(E_{\omega_{0}}(\mu)_{\mid \mu}=-\varphi\right.$. Moreover, since clearly $d\left(D_{d V}(\mu)\right)_{\mid \mu}=\log (\mu / d V)$ differentiating the map $\varphi \mapsto \omega_{\varphi}^{n}$ from $\mathcal{H}\left(X, \omega_{0}\right)$ into $\mathcal{M}_{1}(X)$ gives, using the chain rule, $d\left(F_{\beta}\left(\omega_{\varphi}^{n}\right)\right)_{\mid \varphi} /(n-1)=$

$$
=\omega_{\varphi}^{n-1} \wedge \frac{i}{\pi} \partial \bar{\partial}\left(-\varphi+\beta^{-1} \log \left(\omega_{\varphi}^{n} / d V\right)=\omega_{\varphi}^{n-1} \wedge\left(-\omega_{\varphi}-\alpha-\beta^{-1} \operatorname{Ric} \omega_{\varphi}\right)=\right.
$$

which proves the twisted generalization of formula 4.15 (with $C=1$ ).

Remark 4.13. In the case when $L$ is ample an alternative proof of the fact that $\omega_{\beta}^{n}$ minimizes $F_{\beta}$ on $\mathcal{M}_{1}(X)$ can be given by using that $\omega_{\beta}$ is a critical point of $\kappa$ and hence, by convexity, minimizes $\kappa$ on $\mathcal{H}\left(X, \omega_{0}\right)$. Accordingly, the Calabi-Yau isomorphism $\omega \mapsto \omega^{n}$ shows that $\omega_{\beta}^{n}$ minimizes the restriction of $F_{\beta}$ to the subspace of all volume forms in $\mathcal{M}_{1}(X)$. However, showing that the infimum of $F_{\beta}$ over all of $\mathcal{M}_{1}(X)$ coincides with the infimum over the subspace of volume forms requires the following non-trivial fact: any $\mu$ such that $E(\mu)<\infty$ can be written as a weak limit of volume forms $\mu_{j}$ such that $E\left(\mu_{j}\right) \rightarrow E(\mu)$ and $D_{d V}\left(\mu_{j}\right) \rightarrow D_{d V}(\mu)$ (see [15] where more general results are obtained).

\section{CANONiCAL RANDOM POINT PROCESSES ON VARIETIES OF POSITIVE Kodaira DIMENSION AND LOG PAIRS}

5.1. The case of a klt pair $(X, D)$ of log general type. Assume given a smooth log pair $(X, D)$, where $D$ is klt $\mathbb{Q}$-divisor and assume that $(X, D)$ is of log general type, i.e. $L:=K_{X}+D$ is big. To the pair $(X, D)$ we may attach a canonical sequence of random point processes on $X$ as follows. Fix a smooth metric on $L$ represented by a weight $\phi_{0}$. It determines a singular volume form $\mu_{\left(\phi_{0}, D\right)}$ locally represented as

$$
\mu_{\left(\phi_{0}, D\right)}=e^{\phi_{0}-\phi_{D}} i d z \wedge d \bar{z}
$$

where $i d z \wedge d \bar{z}$ is a short hand for the local Euclidean volume form determines by the local holomorphic coordinates $z$ and $\phi_{0}$ is the corresponding local representation of the weight. Then it if follows immediately from the definitions that the probability measure in formula 4.5 determined by the triple $\left(\mu, \phi_{0}, \beta\right)=\left(\mu_{\left(\phi_{0}, D\right)}, \phi_{0}, 1\right)$ is independent of $\phi_{0}$ and thus canonically attached to $(X, D)$. In fact, it coincides with the probability measure of the canonical random point defined in the introduction of the paper. The point is that if $s_{k}$ is a holomorphic section of $k\left(K_{X}+D\right)$ then the measure

$$
\left\|s_{k}\right\|_{k \phi_{0}}^{2 / k} \mu_{\left(\phi_{0}, D\right)}=\left\|s_{k}\right\|_{k \phi_{0}}^{2 / k} \mu_{\left(\phi_{0}, D\right)}=\left|s_{k}\right|^{2 / k} e^{-\phi_{D}} i d z \wedge d \bar{z},
$$

is clearly independent of $\phi_{0}$.

If $u_{K E}$ is the solution of the corresponding Monge-Ampère equation 4.9 (with $\theta=d d^{c} \phi_{0}$ and $\left.\mu_{0}=\mu_{\left(\phi_{0}, D\right)}\right)$ then it will sometimes be convenient to rewrite the equation in terms of the corresponding weight $\phi_{K E}:=\phi_{0}+u_{K E}$ :

$$
\left(d d^{c} \phi_{K E}\right)^{n}=e^{\phi_{K E}-\phi_{D}} i d z \wedge d \bar{z}
$$

Its curvature current $\omega_{K E}:=d d^{c} \phi_{K E}\left(=\theta+d d^{c} u_{K E}\right)$ satisfies the following log Kähler-Einstein equation associated to $(X, D)$ :

$$
\operatorname{Ric} \omega_{K E}=-\omega_{K E}+[D]
$$

where Ric $\omega$ denotes the Ricci curvature of $\omega$ viewed as a current on $X$. See [14] for the precise meaning of the previous equation in the general setting when $K_{X}+D$ is merely assumed big. Anyway, for $K_{X}+D$ semi-ample and big (or nef and big) 
it was shown in 12 that the solution $\omega$ is smooth on the log regular locus (i.e. on $X-D$ ) and defines a bona fide Kähler-Einstein metric there and its potential $u_{K E}$ is globally continuous on $X$. Moreover, in the case when $K_{X}+D$ is ample the current $\omega$ globally defines a singular Kähler-Einstein metric with edge-cone singularities along $D$ (see [40, 22]). Anyway, in the present setting will not need any regularity properties of $\omega_{K E}$.

The free energy functional

$$
F=E_{d d^{c} \phi_{0}}+D_{\mu_{\left(\phi_{0}, D\right)}} .
$$

determined by the back-ground data $\left(\mu, \phi_{0}, \beta\right)=\left(\mu_{\left(\phi_{0}, D\right)}, \phi_{0}, 1\right)$ is independent of $\phi_{0}$ modulo an additive constant (as follows from the transformation property pointed out in Remark 4.12). In fact, when $K_{X}+D$ is ample the functional $F$ corresponds to the log version $\kappa_{(X . D)}$ of Mabuchi's K-energy functional in the sense that

$$
F\left(\frac{\omega^{n}}{V}\right)=\kappa_{(X . D)}(\omega)
$$

(we recall that $\kappa_{(X . D)}$ is also only defined up to an additive constant). This can be seen as a generalization of a formula of Tian and Chen for the K-energy (see [5, 12] and references therein).

Theorem 5.1. Let $(X, D)$ be a smooth klt pair of general type. Then the empirical measures of the corresponding canonical random point processes on $X$ converge in probability towards the normalized volume form $d V_{K E}$ of the Kähler-Einstein metric on $(X, D)$. More precisely, the laws of the empirical measures satisfy a large deviation principle with speed $N_{k}$ and rate functional $I(\mu):=F(\mu)-C$, where $C=F\left(d V_{K E}\right)$ and $\kappa_{(X . D)}(\omega):=I\left(\omega^{n} / V\right)$ coincides with Mabuchi's (log) K-energy of $\omega$ normalized so that $\kappa_{(X . D)}\left(\omega_{K E}\right)=0$.

Proof. Setting $\left(\mu, \phi_{0}, \beta\right)=\left(\mu_{\left(\phi_{0}, D\right)}, \phi_{0}, 1\right)$ this is a direct consequence of Theorem 4.11.

As a rather direct consequence of the previous theorem we get the following

Corollary 5.2. Let $(X, D)$ be a smooth klt pair of general type. Then the first correlation measures $\nu_{k}:=\int_{X^{N-1}} \mu^{\left(N_{k}\right)}$ of the canonical point processes define a sequence of canonical measures on $X$ converging weakly to $d V_{K E}$. Moreover, the curvature forms of the corresponding metrics on $K_{X}$ defined by the sequence $\nu_{k}$ converge weakly to the unique Kähler-Einstein metric $\omega_{K E}$ on $X$.

Proof. First observe that, by definition, the one point-correlation measure we may be written as

$$
\mu_{1}^{\left(N_{k}\right)}=e^{\phi_{k}} i d z \wedge d \bar{z}=e^{u_{k}} d V
$$

where $\phi_{0}$ is a fixed smooth weight on $K_{X}, d V=\mu_{\phi_{0}}$ and $u_{k} \in P S H(X, \theta)$, for $\theta=$ $d d^{c} \phi_{0}$. In particular, $\int_{X} e^{u_{k}} d V=1$ and hence by Jensen's inequality, $\sup _{X} u_{k} \leq C_{0}$. But, by standard compactness results for $\theta$-psh functions [33, it follows that either $u_{k}$ converges in $L^{1}(X)$ towards some $u \in P S H(X, \theta)$, or there is a subsequence $u_{k_{j}}$ such that $u_{k_{j}} \rightarrow-\infty$ uniformly. But the latter alternative is not compatible with the condition $\int_{X} e^{u_{k}} d V=1$ and hence $u_{k} \rightarrow u$ in $L^{1}(X)$. Equivalently, this means that $\phi_{k} \rightarrow \phi$ in $L_{l o c}^{1}$ where $\phi$ is a weight on $K_{X}$ with positive curvature current. On the other hand, by the previous theorem $\mu_{1}^{\left(N_{k}\right)} \rightarrow e^{\phi_{K E}} i d z \wedge d \bar{z}$, where 
$d d^{c} \phi_{K E}=\omega_{K E}$ (compare Lemma 4.1). But, since a subsequence of $\phi_{k}$ converges a.e. on $X$ it then follows that $\phi_{K E}=\phi$. In particular, $d d^{c} \phi_{k} \rightarrow d d^{c} \phi_{K E}=\omega_{K E}$ weakly and that concludes the proof.

\subsection{Varieties of positive Kodaira dimension.}

5.2.1. Birational setup. Let us start by recalling the standard setup in birational geometry. Let $X$ and $X^{\prime}$ be (normal) projective varieties. A rational mapping $F$ from $X$ to $X^{\prime}$, denoted by a dashed arrow $X \rightarrow X^{\prime}$, is defined by a morphism $F: U \rightarrow X^{\prime}$ from a Zariski open subset $U$ of $X$. It is called birational if it has an inverse. Then there is a maximal Zariski open subset $U \subset X$, where $F$ defines a welldefined isomorphism onto its image (the complement of $U$ is called the exceptional locus of $F$ ). Given a rational mapping $F$ from $X$ to $X^{\prime}$ and a probability measure $X$ which is is absolutely continuous with respect to Lebesgue measure, we can define $F_{*} \mu$ by pushing forward the restriction of $\mu$ to any Zariski open subset $U$ where $F$ is well-defined and pull-backs of such measures can be similarly defined. If $F: X \rightarrow X^{\prime}$ is birational then there exists a non-singular variety $Z$ and birational morphisms $f: Z \rightarrow X$ and $f^{\prime}: Z \rightarrow X^{\prime}$ such that $f^{\prime}=F \circ f$ (in fact, $f$ and $f^{\prime}$ can even be obtained as a sequence of blow-ups and blow-downs respectively). The Kodaira dimension $\kappa(X)$ of an $n$-dimensional (say non-singular) variety $X$ is the birational invariant defined as the smallest number $\kappa \in\{-\infty, 0,1 ., \ldots, n\}$ such that $N_{k}=O\left(k^{\kappa}\right)$, where $N_{k}$ denotes the $k$ th plurigenus of $X$, i.e. the dimension of $H^{0}\left(X, k K_{X}\right)$. In the strictly positive case $\kappa(X)$ may be equivalently defined as the the dimension of the image of $X$ under the $k$-th canonical rational mappings

$$
F_{k}: X \rightarrow \mathbb{P}^{*} H^{0}\left(X, k K_{X}\right), \quad Y_{k}:=\overline{F_{k}(X)}, \quad x \mapsto\left\{s_{k} \in H^{0}\left(X, k K_{X}\right): s_{k}(x)=0\right\}
$$

where here and subsequently $k$ stands for a sufficiently large, or sufficiently divisible, positive integer. By construction $k K_{X}$ is trivial along the fibers of $F_{k}$. Next, we recall that by classical results of Iitaka there exist non-singular varieties $X^{\prime}$ and $Y^{\prime}$ and a subjective morphism $F$ with connected fibers:

$$
F: X^{\prime} \rightarrow Y^{\prime}
$$

such that $X^{\prime}$ and $Y^{\prime}$ are birational to $X$ and $Y$, respectively and such that $F$ is conjugate to $F_{k}$. The fibration defined by $F$ is uniquely determined up to birational equivalence and usually referred to as the Iitaka fibration. A very general fiber of the fibration has vanishing Kodaira dimension.

Finally, it should be pointed out that by the deep results in [19, 42], proved in the context of the MMP, the canonical ring $R(X):=\bigoplus_{k \in \mathbb{N}} H^{0}\left(X, k K_{X}\right)$ of any non-singular projective variety $X$ is finitely generated. In particular, $Y_{k}$ (as defined above) is, for $k$ sufficiently divisible, independent of $k$ (up to isomorphism) and coincides with the canonical model of $X$ (i.e. the Proj of $R(X)$ ). But this information will not be needed for our arguments.

5.2.2. Canonical point processes on varieties of positive Kodaira dimension. Let us now consider a non-singular variety $X$ of positive Kodaira dimension (there is also a logarithmic version of this setup concerning klt pairs $(X, D)$, but for simplicity 
we will assume that $D=0) 2$ On such a variety $X$ we can define the canonical random point processes just as in section 5.1 (since $N_{k}>0$ for $k$ large).

Proposition 5.3. The canonical random point processes attached to a variety $X$ of positive Kodaira dimension are birationally invariant in the sense that if $F$ : $X \rightarrow X^{\prime}$ is a birational mapping, then the canonical probability measures on $X^{N_{k}}$ and $X^{\prime N_{k^{\prime}}}$ are invariant under $F_{*}$.

Proof. This follows from the usual proof of the birational invariance of the spaces $H^{0}\left(X, k K_{X}\right)$. Indeed, $F$ defines an isomorphism from $U$ in $X$ to $U^{\prime}$ in $X^{\prime}$, where $U$ has codimension at least two. Hence, by the usual unique extension properties of holomorphic sections $F_{\mid V}^{*}$ induces an isomorphism between $H^{0}\left(X, k K_{X}\right)$ and $H^{0}\left(X^{\prime}, k K_{X^{\prime}}\right)$, which (by the change of variables formula) respects the measure $\left(S_{k} \wedge \overline{S_{k}}\right)^{1 / k}$ defined by an element $S_{k} \in H^{0}\left(X, k K_{X}\right)$. Applying this argument on the products $X^{N_{k}}$ and $X^{\prime N_{k}}$ then concludes the proof.

When studying the random point processes on $X$ we may without loss of generality, by the previous proposition, assume that there is morphisms $F$ of $X$ to the base $Y$ of the Iitaka fibration. There is a canonical family of relative measures $\mu_{X / Y}$ defined over an open dense subset $Y_{0}$ of $Y$, such that $Y-Y_{0}$ is a null set, defined a follows. First, by the construction of the Iitaka fibration, we may assume that $F$ is a submersion over some open dense subset $Y_{0}$ of $Y$ and that $H^{0}\left(X_{y}, k K_{X_{y}}\right)$ is one-dimensional for $y \in Y_{0}$. Letting $\Omega_{y}^{(k)}$ denote a generator of the latter one-dimensional vector space,

$$
\left(\mu_{X / Y}\right)_{y}:=\left(\Omega_{y}^{(k)} \wedge \overline{\Omega_{y}^{(k)}}\right)^{1 / k} / \int_{X_{y}}\left(\Omega_{y}^{(k)} \wedge \overline{\Omega_{y}^{(k)}}\right)^{1 / k}
$$

is a probability measure on $X_{y}$ which is independent of the generator and of $k$ (since $\left(\Omega_{y}^{(k)}\right)^{\otimes m}$ generates $\left.H^{0}\left(X_{y}, k m K_{X_{y}}\right)\right)$ and it defines a smooth family of relative $(n-\kappa, n-\kappa)-$ forms over $Y_{0}$. Let us also introduce some further notation: if $\nu_{Y}$ is a measure on the base $Y$ which is absolutely continuous with respect to Lebesgue measure, then we will write $F^{*} \nu_{Y} \wedge \mu_{X / Y}$ for the measure on $X$ defined as a fiberproduct, i.e. if $u$ is a smooth function on $X$ then

$$
\int_{X} F^{*} \nu_{Y} \wedge \mu_{X / Y} u:=\int_{Y_{0}}\left(\int_{X_{y}} u \mu_{X / Y}\right) \nu_{Y}
$$

(which is independent of the choice of $Y_{0}$ since the complement is a null set).

Lemma 5.4. Let $X$ be a variety of positive Kodaira dimension and assume that the Iitaka fibration $F: X \rightarrow Y$ is a morphism and that the branch locus $Y-Y_{0}$ is equal to the support of a divisor $D$ in $Y$ with normal crossings. Then there exists a line bundle $L_{X / Y}$ over $Y$ equipped with a (singular) metric whose weight will be denoted by $\phi_{H}$, with the property that $K_{Y}+L_{X / Y}$ is big and for any $S_{k} \in H^{0}\left(X, k K_{X}\right)$ there exists a unique $s_{k} \in H^{0}\left(Y, k\left(K_{Y}+L_{X / Y}\right)\right.$ such that

$$
\left(S_{k} \wedge \overline{S_{k}}\right)^{1 / k}=F^{*}\left(\left(s_{k} \wedge \overline{s_{k}}\right)^{1 / k} e^{-\phi_{H}}\right) \wedge \mu_{X / Y}
$$

\footnotetext{
${ }^{2}$ More generally, the results will apply to $X$ a possibly singular normal variety, by defining the corresponding probability measures on the regular locus of $X$ and using the birational invariance below to replace $X$ with any resolution.
} 
over $Y_{0}$. The weight $\phi_{H}$ is smooth on $Y_{0}$ and locally around any given point in $Y-Y_{0}$

$$
\phi_{H}=-q \log \log \left(\left|s_{D}\right|^{-2}\right)+\log \left|s_{D_{X / Y}}\right|^{2}+O(1)
$$

for some positive number $q$, where $D_{X / Y}$ is a klt divisor whose support coincides with $D$. The line bundle $L_{X / Y}$ will be referred to as the Hodge line bundle and $\phi_{H}$ as the weight of the Hodge metric.

Proof. By assumption the morphism $F$ restricts to define a submersion $\pi: X_{0} \rightarrow Y_{0}$ between Zariski open subsets. The (tautological) decomposition $K_{X}=F^{*} K_{Y}+$ $K_{X / Y}$ restricted over $Y^{0}$ gives

$$
k K_{X_{0}}=F^{*} k\left(K_{Y_{0}}+F^{*} L_{0}\right),
$$

where $L_{0}:=\pi_{*}\left(K_{X_{0} / Y_{0}}\right)$. The latter direct image sheaf is defined as a $\mathbb{Q}$-line bundle over $Y_{0}: F_{*}\left(K_{X_{0} / Y_{0}}\right)=\frac{1}{k} F_{*}\left(k K_{X_{0} / Y_{0}}\right)$ for any fixed $k$ which is sufficiently large. Concretely, $k L_{0}$ is locally trivialized by $\Omega_{y}^{(k)}$, where $\Omega_{y}^{(k)}$ is as in formula 5.4 We equip the direct image line bundle $L_{0}$ with the canonical $L^{2}$-metric, usually referred to as the Hodge metric. Concretely, the $k$ tensor power of the letter metric is defined by $\left\|\left(\Omega_{y}^{(k)}\right)\right\|^{2}:=\left(\int_{X_{y}}\left(\Omega_{y}^{(k)} \wedge \overline{\Omega_{y}^{(k)}}\right)^{1 / k}\right)^{k}$, i.e the local weight $\phi_{H}$ for $L_{0}$ determined by the trivialization (multi-) section $\left(\Omega_{y}^{(k)}\right)^{1 / k}$ is given by

$$
\left.\phi_{H}(y):=-\log \left\|\left(\Omega_{y}^{(k)}\right)^{1 / k}\right\|^{2}=-\log \int_{X_{y}}\left(\Omega_{y}^{(k)} \wedge \overline{\Omega_{y}^{(k)}}\right)^{1 / k}\right)
$$

Next, fixing $x \in X_{0}$ we take a small neighborhood $V$ of $y_{0}:=F\left(x_{0}\right)$ and local holomorphic coordinates $w$ centered at $y_{0}$ and set $d w:=d w_{1} \wedge \cdots \wedge d w_{\kappa}$. Then the restriction of $S_{k} \in H^{0}\left(X, k K_{X}\right)$ to $U:=F^{-1}(V)$ may be written as $S_{k}=$ $f_{k}(w) \Omega_{y}^{(k)} \otimes d w^{\otimes k}$ for a local holomorphic function $f_{k}(w)$ on $V$ (which transforms as a section of $\left.k L_{0} \rightarrow Y_{0}\right)$ and

$$
\left(S_{k} \wedge \overline{S_{k}}\right)^{1 / k}=\left|f_{k}(w)\right|^{2 / k} d w \wedge d \bar{w} \wedge \Omega_{y}^{(k)} \wedge \overline{\Omega_{y}^{(k)}}=\left|f_{k}(w)\right|^{2 / k} e^{-\phi_{H}(w)} d w \wedge d \bar{w} \wedge \mu_{X / Y}
$$

where $\mu_{X / Y}$ is the relative probability measure defined in formula [5.4. Since $x$ was an arbitrary point in $X_{0}$ this proves the relation 5.5 over $Y_{0}$ if $s_{k}$ is taken in $H^{0}\left(Y_{0}, k\left(K_{Y}+L_{0}\right)\right.$.

Next, we will give the construction of the line bundle $L_{X / Y}$ extending $L_{0}$ and show that $s_{k}$ above can be taken as the restriction to $Y_{0}$ of an element in $H^{0}\left(Y, k\left(K_{Y}+\right.\right.$ $\left.L_{X / Y}\right)$. First, following Fujino-Mori 30, we may assume that the double dual of the torsion free sheaf $\pi_{*}\left(k K_{X / Y}\right) / k$ is a well-defined $\mathbb{Q}$-line bundle and set $L_{X / Y}:=L_{0}$. The canonical bundle formula of Fujino-Mori (see Prop 2.2. in [30]) says that

$$
K_{X}+B_{-}=\pi^{*}\left(K_{Y}+L_{X / Y}\right)+B_{+}
$$

where $B_{ \pm}$are effective $\mathbb{Q}$-divisors (supported in $\left.Y-Y_{0}\right)$ such that $\operatorname{codim} F\left(\operatorname{supp}\left(B_{-}\right)\right) \geq$ 2 and $F_{*}\left(\mathcal{O}\left(k B_{+}\right)=\mathcal{O}_{Y}\right.$. This formula implies that

- If $S_{k} \in H^{0}\left(X, k K_{X}\right)$, then the restriction of $S_{k}$ to $X_{0}$ may be written as $S_{k \mid X_{0}}=F^{*} s_{k \mid Y_{0}}$ for a unique section $s_{k} \in H^{0}\left(Y, k\left(K_{Y}+L_{X / Y}\right)\right)$. 
Indeed, by the canonical bundle formula and the property of $B_{+}$, the restriction of $S_{k}$ to $Y-\operatorname{supp}\left(B_{-}\right)$may be written as $S_{k}=F^{*} s_{k} \otimes s_{B_{+}}^{\otimes k}$ for a unique $s_{k} \in$ $H^{0}\left(Y-F\left(\operatorname{supp}\left(B_{-}\right)\right), k K_{Y}\right)$. But since $\operatorname{codim} F\left(\operatorname{supp}\left(B_{-}\right)\right) \geq 2$ the section $s_{k}$ extends to a unique element in $H^{0}\left(Y, k\left(K_{Y}+L_{X / Y}\right)\right)$. Since $\operatorname{supp}\left(B_{-}\right) \subset Y-Y_{0}$ this proves the point above. Note that this is essentially the same argument as the one used in [30] to prove that $H^{0}\left(X, k K_{X}\right)=H^{0}\left(X, k\left(K_{Y}+L_{X / Y}\right)\right.$ (see the proof of Theorem 4.5 in op.cit.) and it also shows that $K_{Y}+L_{X / Y}$ is big (since $N_{k} \sim k^{\kappa}$ ). Finally, let us briefly recall the proof of the singularity structure of the Hodge metric on $L_{X / Y}$ in a neighborhood of a point contained in $Y-Y_{0}$, which follows from Tsuji's argument in [48. First, as shown in 30 may assume that $L_{X / Y}=M_{X / Y}+D_{X / Y}$, where $M_{X / Y}$ (" the semi-stable part") is a nef line bundle on $Y$ and $D_{X / Y}$ (the "discriminant part") is a klt divisor on $Y$. By the work of Kawamata and Schmidt on variations of Hodge structures (see [48] and references therein) the lines bundles $M_{X / Y}$ and $D_{X / Y}$ contribute over $Y-Y_{0}$ to the first and second term in the decomposition [5.6 of the weight $\phi_{H}$ defined with respect to a given trivialization of $L_{X / Y}$ over a neighborhood of a point in $Y-Y_{0}$.

Next, let us recall the definition of the (singular) canonical metric $\omega_{Y}$ on the base of the base $Y$ of the Iitaka fibration (which is a birational invariant). For our purposes it will be enough to define it in the case when $X$ fibers over $Y$ as in the previous lemma. Then we define $\omega_{Y} \in c_{1}\left(K_{Y}+L_{X / Y}\right)$ as $\omega_{Y}=d d^{c} \phi_{Y}$, where $\phi_{Y}$ is the weight of a (possible singular) positively curved metric $\phi_{Y}$ on $K_{X}+L_{X / Y}$ defined as the unique finite energy weight $\phi$ on the big line bundle $K_{X}+L_{X / Y}$ solving

$$
M A(\phi)=e^{\phi-\phi_{H}} i d w \wedge d \bar{w} .
$$

(which is an equation of the form appearing in Prop 4.9) . Note that by the previous lemma we have that locally $e^{-\phi} \in L_{l o c}^{p}$ for some $p>1$ and hence by the Kolodziej type estimates in 20] $\phi$ has minimal singularities. We may hence define $\omega_{Y}$ equivalently as the unique current in $c_{1}\left(K_{Y}+L_{X / Y}\right)$ with minimal singularities such that

$$
\text { Ric } \omega_{Y}=-\omega_{Y}+\omega_{W P}+[\Delta] \text {, }
$$

where $\Delta$ is the klt divisor of Fujino-Mori (the "discriminant divisor") supported on the branch locus in $Y$ and $\omega_{W P}$ is equal to $1_{Y_{0}} \omega_{W P}$ where $\omega_{W P}$ is the generalized Weil-Petersson type metric of the fibration over $Y_{0}$ (compare [45]). It may be defined as the curvature form of the Hodge metric on $L_{X / Y} \rightarrow Y_{0}$. Alternatively, we note that arguing as in the beginning of the proof of the previous lemma gives the following equivalent equation for $\phi_{Y}$, where the pull-back and push-forward is defined over $Y_{0}$ (and then extended by zero):

$$
M A\left(\phi_{Y}\right)=F_{*}\left(e^{F^{*} \phi_{Y}} d z \wedge d \bar{z}\right)
$$

(where the right hand side is defined with respect to suitable local coordinates $(z, w)$ on $X$ respecting the fibration). Finally, we define the canonical probability measure $\mu_{X}$ on $X$ of Song-Tian-Tsuji as

$$
\mu_{X}:=F^{*} \mu_{Y} \wedge \mu_{X / Y}
$$


Equivalently, $\mu_{X}=F^{*} \omega_{Y}^{\kappa} \wedge \omega_{C Y}^{n . \kappa}$, where $\omega_{C Y}$ denotes a family of Ricci flat Kähler metrics defined over the very general Calabi-Yau fibers and the metrics are normalized to have unit-volume (abusing terminology slightly the term Calabi-Yau refers to a variety of zero Kodaira dimension).

Theorem 5.5. Let $X$ be projective variety of positive Kodaira dimension. Then the empirical measures of the canonical random point processes on $X$ converge in probability towards the canonical probability measure $\mu_{X}$ of Song-Tian-Tsuji.

Proof. By the previous lemma we can write $\mu^{\left(N_{k}\right)}=F^{*} \mu_{Y}^{\left(N_{k}\right)} \wedge \mu_{X / Y}^{\otimes N_{k}}$ where $\mu_{Y}^{\left(N_{k}\right)}$ is defined with respect to the big line bundle $K_{Y}+L_{X / Y} \rightarrow Y$ equipped, where $L_{X / Y}$ is equipped with the Hodge metric. In particular, the $j$-point correlation measures $\left(\mu^{\left(N_{k}\right)}\right)_{j}$ are given by

$$
\left(\mu^{(N)}\right)_{j}:=\int_{X^{N-j}} \mu^{(N)}=F^{*}\left(\mu_{Y}^{\left(N_{k}\right)}\right)_{j} \wedge \mu_{X / Y}^{\otimes j}
$$

Next we note that we may proceed as in the proof of Theorem 4.11 to see that the empirical measures of $\mu_{Y}^{\left(N_{k}\right)}$ converge in probability towards $\mu_{Y}$ (and even with with a LDP), which implies that

$$
\left(\mu_{Y}^{\left(N_{k}\right)}\right)_{j} \rightarrow \mu_{Y}^{\otimes j}
$$

(compare Cor ??). Indeed, fixing smooth Hermitian metrics (weights) $\phi$ and $\phi_{0}$ on $K_{Y}$ and $L_{X / Y}$ the measure $\left(s_{k} \wedge \overline{s_{k}}\right)^{1 / k} e^{-\phi_{H}}$ defined by an element $s_{k} \in$ $H^{0}\left(Y, k\left(K_{Y}+L_{X / Y}\right)\right.$ may be written as $\left\|s_{k}\right\|^{2} \mu_{\phi} e^{-\left(\phi_{H}-\phi_{0}\right)}$ and by the previous lemma

$$
\mu_{\phi} e^{-\left(\phi_{H}-\phi_{0}\right)}=e^{-v} \mu_{\Delta}:=\mu_{0}
$$

for a klt divisor $\Delta$ where $v$ is upper semi-continuous and such that $e^{-v} \mu_{\Delta}$ is a finite measure (even with an $L^{p}$-density for some $p>1$ ). But then we may proceed precisely as in the proof of Theorem 4.11 the argument for the upper bound works the same for any finite measure $\mu_{0}$ and for the proof of the lower bound we can take a sequence $v_{j}$ of continuous functions decreasing to $v$ and replace $\mu_{0}$ with $\mu_{j}:=e^{-v_{j}} \mu_{\Delta}$. Then we let $j \rightarrow \infty$ in the end of the argument, just as before and obtain the desired convergence in probability towards the deterministic measure $\mu_{Y}$ satisfying $\mu_{Y}=M A\left(\phi_{Y}\right)$, where $M A\left(\phi_{Y}\right)=e^{\phi_{Y}-\phi_{H}} i d w \wedge d \bar{w}$, as desired.

Finally, combining the previous convergence with Sanov's theorem gives

$$
\left(\mu^{(N)}\right)_{j} \rightarrow F^{*}\left(\mu_{Y}^{\otimes j}\right) \wedge \mu_{X / Y}^{\otimes j}=\left(F^{*}\left(\mu_{Y}\right) \wedge \mu_{X / Y}\right)^{\otimes j},
$$

which equivalently means that the canonical empirical measures on $X$ converge in probability towards $\left(F^{*}\left(\mu_{Y}\right) \wedge \mu_{X / Y}\right.$, as desired.

Corollary 5.6. Let $X$ be projective variety of positive Kodaira dimension. Then the currents

$\omega_{k}:=\frac{i}{2 \pi} \partial \bar{\partial} \log \int_{X^{N_{k}-1}}\left|\left(\operatorname{det} S^{(k)}\right)\left(\cdot, z_{1}, \ldots, z_{N_{k}-1}\right)\right|^{2 / k} d z_{1} \wedge d \bar{z}_{1} \wedge \cdots \wedge d z_{N_{k}-1} \wedge d \bar{z}_{N_{k}-1}$

converge, as $k \rightarrow \infty$, weakly towards a canonical positive current in $c_{1}\left(K_{X}\right)$ which, on a Zariski open subset coincides with $F^{*} \omega_{Y}$, i.e. the pull-back of the canonical metric on the base of the Iitaka fibration. 
Proof. Arguing exactly as in the proof of Cor 5.2 gives that $\omega_{k} \rightarrow d d^{c} \log \left(\mu_{X}\right)$. But specializing formula 5.9 to $X_{0}$ over $Y_{0}$ gives that $d d^{c} \log \left(\mu_{X}\right)=d d^{c} F^{*} \omega_{Y}+0$ on $X_{0}$, using that $d d^{c} \log \left|\Omega_{y}^{(k)}\right|^{2}=0$ and that the terms involving $d d^{c} \phi_{H}$ cancel (as is seen by working on a local set $U$ in $X_{0}$ as in the beginning of the proof of the previous lemma).

Remark 5.7. If $K_{X}$ is semi-ample we can take $F$ to be the morphism defined by the canonical map at some fixed level $k$ so that $Y:=X_{c a n}$ is the canonical model of $X$. Then we can define $\omega_{\text {can }}$ as $\omega_{c a n}:=d d^{c} \phi_{c a n}$ where $\phi_{c a n}$ is the unique (locally bounded) positively curved metric on $\mathcal{O}(1)_{\mid X_{c a n}}$ solving the equation 5.8 (using that $\mathcal{O}(1)$ is naturally isomorphic to $K_{Y}+L_{X / Y}$ over $\left.Y_{0}\right)$. The metric $\omega_{\text {can }}$ thus defined yields a canonical Kähler metric in $c_{1}\left(\mathcal{O}(1)_{\mid X_{c a n}}\right)$ which, by the uniqueness argument in [45] coincides with the canonical metric constructed in [45]. Accordingly, the limiting current obtained in the previous corollary coincides with $F^{*} \omega_{\text {can }}$ on $X_{0}$ and hence everywhere since the currents are elements in the same cohomology class $c_{1}\left(K_{X}\right)$. In this setting the previous corollary is thus analogous to the convergence result for the Kähler-Ricci flow for a variety with $K_{X}$ semi-ample obtained in [45].

\section{FAnO MANifolds AND GiBbs Stability}

In this section we will outline a conjectural general picture concerning the the case when $\beta=-1$ in the Kähler-Einstein equation, i.e. the case of Kähler-Einstein metrics with positive Ricci curvature. In other words, this the case when the dual $-K_{X}$ of the canonical line bundle is ample, which means that $X$ is a Fano manifold. We will establish a weak form of the conjecture, but we leave the general case for the future.

If a Kähler-Einstein metric exists on a Fano manifold $X$ then, by the BandoMabuchi theorem, it is uniquely determined modulo the action of automorphism group generated by holomorphic vector fields on $X$. But in general there are obstructions to the existence of a Kähler-Einstein metric on $X$ and according to the Yau-Tian-Donaldson conjecture $X$ admits a Kähler-Einstein metric precisely when $X$ is K-polystable. This latter notion of stability is of an algebro-geometric nature and can be formulated as an asymptotic version of stability in the sense of Geometric Invariant Theory (GIT). Recently, the conjecture was finally settled by ChenDonaldson-Sun [23] (see also [4]). Here we will introduce a probabilistic/statistical mechanical version of the Yau-Tian-Donaldson where the notion of K-stability is replaced by a notion that we will call Gibbs stability. To explain this first observe that to be able to define an analog of the probability measure appearing in formula 1.2 in the introduction of the paper to the Fano setting we have to replace $K_{X}$ with its dual $-K_{X}$ to ensure the existence of holomorphic sections. But this forces us to replace the exponent $1 / k$ with $-1 / k$, in order to get a well-defined density on $X^{N_{k}}$. However, there is then no guarantee that the corresponding normalization constant

$$
Z_{N_{k}}:=\int_{X^{N_{k}}}\left|\left(\operatorname{det} S^{(k)}\right)\left(z_{1}, \ldots, z_{N_{k}}\right)\right|^{-2 / k} d z_{1} \wedge d \bar{z}_{1} \wedge \cdots \wedge d z_{N_{k}} \wedge d \bar{z}_{N_{k}}
$$

is finite, since the corresponding integrand is singular along the zero-locus of $\operatorname{det} S^{(k)}$. Accordingly, we say that a Fano manifold $X$ is Gibbs stable at level $k$ if $Z_{N_{k}}$ is finite (which is independent of the choice of generator $\operatorname{det} S^{(k)}$ ) and asymptotically Gibbs stable if it is Gibbs stable at level $k$ for any $k$, sufficiently large. 
Conjecture 6.1. Let $X$ be Fano manifold. Then $X$ admits a unique KählerEinstein metric $\omega_{K E}$ if and only if $X$ is asymptotically Gibbs stable. Moreover, if $X$ is asymptotically Gibbs stable, then the empirical measures of the corresponding point processes converge in probability towards the normalized volume form of $\omega_{K E}$ and the corresponding canonical sequence of curvature forms $\omega_{k}$ converge weakly towards $\omega_{K E}$.

Here $\omega_{k}$ is defined by

$-\frac{i}{2 \pi} \partial \bar{\partial} \log \int_{X^{N_{k}-1}}\left|\left(\operatorname{det} S^{(k)}\right)\left(\cdot, z_{1}, \ldots, z_{N_{k}-1}\right)\right|^{-2 / k} d z_{1} \wedge d \bar{z}_{1} \wedge \cdots \wedge d z_{N_{k}-1} \wedge d \bar{z}_{N_{k}-1}$.

It follows from applying Berndtsson's positivity of direct images $18 N-1$ times (i.e. for each factor of $X^{N-1}$ ) that $\omega_{k}$ is in fact positive in the sense of currents and hence a Kähler form for $k$ sufficiently large. One can also define a weaker notion of Gibbs stability which takes automorphisms into account, but for simplicity we will focus here on the case when $X$ admits no automorphisms.

Interestingly, the notion of Gibbs stability introduced above can also be given the following purely algebro-geometric formulation in the spirit of the Minimal Model Program: let $\mathcal{D}_{k}$ be the effective divisor in $X^{N_{k}}$ cut out by the section $\operatorname{det} S^{(k)}$. Geometrically, $\mathcal{D}_{k}$ may be represented as the following incidence divisor in $X^{N_{k}}$ :

$$
\mathcal{D}_{k}:=\left\{\left(x_{1}, \ldots x_{N}\right) \in X^{N_{k}}: \exists s \in H^{0}\left(X,-k K_{X}\right): s\left(x_{i}\right)=0, i=1, . ., N_{k}\right\}
$$

Gibbs stability at level $k$ amounts to saying that $\mathcal{D}_{k} / k$ is mildly singular in the sense of MMP (i.e. its singularities are Kawamata Log Terminal) or more precisely that

$$
\operatorname{lct}\left(\mathcal{D}_{k} / k\right)>1
$$

for $k>>1$, where $\operatorname{lct}\left(\mathcal{D}_{k} / k\right)$ denotes the the $\log$ canonical threshold (lct) of the anti-canonical $\mathbb{Q}$-divisor divisor $\mathcal{D}_{k} / k$ on $X^{N_{k}}$. The equivalence with the original definition follows directly from the analytic definition of the lct of a divisor $D=$ $\{s=0\}$ as the sup of all $t$ such that $1 /|s|^{2 t}$ is locally integrable (also using the "openness property" for the lct of algebraic singularities). It also seems natural to say that $X$ is uniformly Gibbs stable, if

$$
\gamma(X):=\liminf _{k \rightarrow \infty} \operatorname{lct}\left(\mathcal{D}_{k} / k\right)>1
$$

There is also an even stronger notion of Gibbs stability which we call strong Gibbs stability and which demands that

$$
\lim _{k \rightarrow \infty} \frac{1}{N_{k}} \log Z_{N_{k}, \beta}<\infty
$$

for some $\beta<1$, where $Z_{N_{k}, \beta}$ is the partition function at inverse temperature $\beta$ (formula 4.6) defined with respect to a metric $\|\cdot\|$ on $-K_{X}$, a volume form $d V$ (for example the one defined by the metric $\|\cdot\|$ ) and the corresponding generator $\operatorname{det} S^{(k)}$.

Proposition 6.2. Suppose that $X$ admits non-trivial holomorphic vector fields. Then $\gamma(X) \leq 1$. More precisely, for $k$ sufficiently large the canonical partition function $Z_{N_{k}}$ is non-finite.

Proof. Assume to get a contradiction that $Z_{N_{k}}$ is finite. Then we may define a probability measure $\nu_{k}$ on $X$ as the corresponding one-point correlation measure. But, by construction, $\nu_{k}$ is invariant under the action of the automorphism group 
of $X$. Moreover, for $k$ sufficiently large $H^{0}\left(X,-k K_{X}\right)$ is basepoint free and hence $\nu_{k}$ then defines a volume form on $X$. But it follows from general principles that if a complex manifold $X$ admits a non-trivial holomorphic vector field $v$ than it cannot admit an automorphism invariant volume form. This is proved in a standard way by taking the Lie derivative of $\nu_{k}$ along the vector field $v$ and using Cartan's formula.

Next, we will show the following weak partial confirmation of the conjecture above.

Theorem 6.3. Suppose that the Fano manifold $X$ is strongly Gibbs stable. Then it admits a unique Kähler-Einstein metric. More generally, the corresponding result holds in the setting of (possibly singular) log Fano varieties.

Proof. Fix a volume form $\mu$ on $X$. By the Gibbs variational principle (i.e. Jensen's inequality)

$$
-\frac{1}{N_{k} \beta} \log Z_{N_{k}, \beta} \leq E^{(N)}\left(\mu^{\otimes N}\right)+\frac{1}{\beta} D_{\mu_{0}}(\mu),
$$

where, for any given symmetric probability measure $\mu_{N}$ on $X^{N}$

$$
E^{(N)}\left(\mu_{N}\right):=\int_{X^{N}} \frac{H^{(N)}}{N} \mu_{N}
$$

i.e. the mean $N$-particle energy. Setting $\beta=-(1+\delta)$ for some $\delta>0$ and using the definition of Gibbs stability thus gives

$$
-(1+\delta) E^{(N)}\left(\mu^{\otimes N}\right)+D_{\mu_{0}}(\mu) \geq-C
$$

We will conclude the proof by observing that

$$
\liminf _{N \rightarrow \infty} E^{(N)}\left(\mu^{\otimes N}\right) \geq E_{\theta}(\mu) .
$$

Accepting this for the moment gives that $(1+\delta) E(\mu)+D_{\mu_{0}}(\mu) \geq-C$, which, by definition, means that the canonical free energy functional $F$ (for $\beta=1$ ) is coercive. But since the latter functional may be identified with Mabuchi's K-energy functional it then follows from a result of Tian that $X$ admits a Kähler-Einstein metric (which by the coercivity has to be unique, since the coercivity rules out automorphisms). More generally, the existence of a minimizer of the functional $F$, satisfying the corresponding Monge-Ampère equations was shown in [5, 12] in various singular settings, in particular in the setting of log Fano varieties [12]. Finally, let us prove the lower bound 6.4 The proof is similar to the upper bound in the proof of Theorem 4.11. By definition, for any given $u \in C^{0}(X)$

$$
E^{(N)}\left(\mu^{\otimes N}\right)=\int_{X^{N}} \frac{H^{(N)}}{N} \mu^{\otimes N}=\int_{X^{N}} \frac{H^{(N)}+u}{N} \mu^{\otimes N}-\int_{X} u \mu
$$

Hence, estimating $\frac{H^{(N)}+u}{N}$ from below, using the first point in Theorem 3.6 and taking the sup over all $u \in C^{0}(X)$ proves 6.4 (also using Prop 3.4 in the last step).

It may very well be that in the end all the notions of Gibbs stability introduced above will turn out to be equivalent. For the moment the author has only been able to prove this in the the first non-trivial setting of one dimensional log Fano manifolds, where the analog of the Conjecture 6.1 indeed holds (the proof will appear in a separate publication). 
It seems also natural to conjecture that the following invariant $\gamma(X)$ defined by 6.3 satisfies

$$
\min \{\gamma(X), 1\}=R(X),
$$

where $R(X)$ is the greatest lower bound on the Ricci curvature [46], i.e. the sup over all $r \in[0,1]$ such that there exists a Kähler form $\omega \in c_{1}\left(-K_{X}\right)$ satisfying Ric $\omega \geq r \omega$. As support for the latter conjecture we note that the inequality $R(X) \geq \min \{\gamma(X), 1\}$ follows from a simple modification of the proof of the previous theorem. Finally, let us point out that these conjectures are related to another conjectural property, namely that the reversed inequality in formula 6.4 holds, i.e. that

$$
\lim _{N \rightarrow \infty} E^{(N)}\left(\mu^{\otimes N}\right)=E_{\theta}(\mu)
$$

for any volume form on $\mu$ (see the discussion in [8, Section 6]). In one dimension this follows from the bosonization formula and it also holds in the toric setting when $\mu$ is a torus invariant measure (as follows form the results in [7]). The validity of formula 6.5 would imply the following approximation result for the Calabi-Yau equation [51]: given a normalized volume form $d V$ on $X$ and an ample line bundle $L \rightarrow X$ the Kähler metrics

$$
\omega_{k}:=\frac{i}{\pi} \partial \bar{\partial} \frac{1}{k} \int_{X^{N_{k}-1}} \log \left|\operatorname{det} S_{k}\left(\cdot, x_{1}, \ldots, x_{N_{k}-1}\right)\right| d V^{\otimes N_{k}-1} \in c_{1}(L)
$$

converge weakly towards the unique Kähler form $\omega$ in $c_{1}(L)$ with volume form $d V$. This conjectural approximation result is the Kähler analogue of the approximation for optimal transport maps obtained in [7] in a convex analytical setting.

Remark 6.4. Since the appearance of the first preprint version of the present paper, where the conjectures above were formulated, there has been some progress that we briefly recall. First, it was shown by Fujita that uniform Gibbs stability implies K-stability and hence by 23 the existence of a Kähler-Einstein metric on $X$, which thus partly confirms Conjecture 6.1 above (when using the notion of uniform Gibbs stability). The proof in 31] proceeds by an elegant algebraic argument using Odaka's formulation of K-stability in terms of equivariant multiplier ideal sheaves together with Mustata's summation formula for multiplier ideal sheaves. More generally, very recently it was shown in 32 that uniform Gibbs stability implies uniform K-stability (and hence the existence of a Kähler-Einstein metric could alternatively be deduced from the recent variational approach to the Yau-Tian-Donaldson conjecture introduced in [17], showing that the existence of a Kähler-Einstein metric on a Fano manifold, not admitting non-trivial holomorphic vector fields, is equivalent to uniform K-stability). Finally, let us also mention that a real (tropical) analog of Conjecture 6.1 in the setting of toric Fano manifolds, where the role of determinants is played by permanents, has been established in [16]. It implies, in particular, the inequality $\min \{\gamma(X), 1\} \leq R(X)$ when $X$ is toric.

\section{REFERENCES}

[1] Aubin, T: Equations du type Monge-Amp'ere sur les vari 'et'es K" ahl'eriennes compactes", Bull. Sc. Math. 102 (1978).

[2] Berman, R.J: Bergman kernels and equilibrium measures for line bundles over projective manifolds. The American J. of Math., Vol 131, Nr. 5, 2009

[3] Determinantal point processes and fermions on complex manifolds: Large deviations and Bosonization. Comm. in Math. Phys. Volume 327, Issue 1 (2014), Page 1-47 
[4] Berman, R.J: Kahler-Einstein metrics emerging from free fermions and statistical mechanics. 22 pages, J. of High Energy Phys. (JHEP), Volume 2011, Issue 10 (2011)

[5] Berman, R.J: A thermodynamical formalism for Monge-Ampere equations, Moser-Trudinger inequalities and Kahler-Einstein metrics. Advances in Math. 1254. Volume: 248. 2013

[6] Berman, R.J: K-polystability of Q-Fano varieties admitting Kahler-Einstein metrics. Inventiones Math. March 2016, Volume 203, Issue 3, pp 973-1025

[7] Berman, R.J: Statistical mechanics of permanents, real-Monge-Ampere equations and optimal transport. arXiv: 1302.4045

[8] Berman, R.J: Large deviations for Gibbs measures with singular Hamiltonians and emergence of Kähler-Einstein metrics. Preprint.

[9] Berman, R.J.; Boucksom, S: Growth of balls of holomorphic sections and energy at equilibrium. Invent. Math. Vol. 181, Issue 2 (2010), p. 337

[10] Berman, R.J.; Boucksom, S; Witt Nyström, D: Fekete points and convergence towards equilibrium measures on complex manifolds. Acta Math. Vol. 207, Issue 1 (2011), 1-27

[11] Berman, R.J; Boucksom, S; Guedj,V; Zeriahi: A variational approach to complex MongeAmpere equations. Publications math. de l'IHÉS (2012): 1-67, November 14, 2012

[12] Berman, R.J; Boucksom,S; Eyssidieu, P; V. Guedj, A. Zeriahi: Kähler-Einstein metrics and the Kähler-Ricci flow on log Fano varieties. Crelle's journal (to appear). arXiv:1111.7158

[13] Berman, R.J; Freixas i Montplet: G: An arithmetic Hilbert-Samuel theorem for singular hermitian line bundles and cusp forms. Compositio Mathematica. Vol. 150/Issue 10 (2014)

[14] Berman, R.J; Guenanica, H: Kähler-Einstein metrics on stable varieties and log canonical pairs. Geometric and Functional Analysis 2014, Volume 24, Issue 6, pp 1683-1730

[15] Berman, R.J; Darvas, T; Lu, C.H: Convexity of the extended K-energy and the large time behaviour of the weak Calabi flow. arXiv:1510.01260

[16] R.J. Berman, Magnus Onnheim: Propagation of chaos, Wasserstein gradient flows and toric Kahler-Einstein metrics. arXiv:1501.07820

[17] Berman, R.J; Boucksom, S; Jonsson, M: A variational approach to the Yau-Tian-Donaldson conjecture. arXiv:1509.04561

[18] Berndtsson, B: Curvature of vector bundles associated to holomorphic fibrations. Annals of Math. Vol. 169 (2009), 531-560

[19] Birkar, C; Cascini, P; Hacon, C.D; McKernan, J: Existence of minimal models for varieties of $\log$ general type. J. Amer. Math. Soc. 23 (2010), no. 2, 405-468

[20] Boucksom, S; Essidieux,P: Guedj,V; Zeriahi: Monge-Ampere equations in big cohomology classes. Acta Math. 205 (2010), no. 2, 199-262.

[21] Caglioti.E; Lions, P-L; Marchioro.C; Pulvirenti.M: A special class of stationary flows for two-dimensional Euler equations: a statistical mechanics description. Communications in Mathematical Physics (1992) Volume 143, Number 3, 501-525

[22] Campana, F; Guenancia, H; Păun, M: Metrics with cone singularities along normal crossing divisors and holomorphic tensor fields. Ann. Sci. Éc. Norm. Supér. (4) 46 (2013), no. 6, 879-916

[23] Chen, X; Donaldson, S; Sun, S: Kahler-Einstein metrics and stability III: Limits as cone angle approaches 2 and completion of the main proof. J. Amer. Math. Soc. 28 (2015), no. 1, 235-278.

[24] Dembo, A; Zeitouni O: Large deviation techniques and applications. Jones and Bartlett Publ. 1993

[25] C Doran, M Headrick, CP Herzog, J Kantor: Numerical Kaehler-Einstein metric on the third del Pezzo. Commun. in Math. Physics, 2008

[26] Donaldson, S. K: Scalar curvature and projective embeddings. I. J. Differential Geom. 59 (2001), no. 3, 479-522

[27] Donaldson, S. K. Some numerical results in complex differential geometry. Pure Appl. Math. Q. 5 (2009), no. 2, Special Issue: In honor of Friedrich Herzebruch. Part 1, 571-618.

[28] Donaldson, S.K.: Kahler metrics with cone singularities along a divisor. Essays in mathematics and its applications, 49-79, Springer, Heidelberg, 2012.

[29] Eyssidieux, P., Guedj, V., Zeriahi, A., Singular K“ahl er-Einstein metrics. J. Amer. Math. Soc. 22 (2009) no. 3, 607-639.

[30] Fujino, O; Mori, S: A canonical bundle formula. J. Differential Geom. 56 (2000), no. 1, $167-188$. 
[31] Fujita, K: On Berman-Gibbs stability and K-stability of X-Fano varieties. Compositio Mathematica 152 (2015) 288-298

[32] Fujita, K; Odaka, Y: On the K-stability of Fano varieties and anticanonical divisors. arXiv:1602.01305

[33] Guedj,V; Zeriahi, A: Intrinsic capacities on compact Kähler manifolds. J. Geom. Anal. 15 (2005), no. 4, 607-639.

[34] Guenancia, H; Păun, M: Conic singularities metrics with prescribed Ricci curvature: the case of general cone angles along normal crossing divisors. http://arxiv.org/abs/1307.6375

[35] Kiessling M.K.H.: Statistical mechanics of classical particles with logarithmic interactions, Comm. Pure Appl. Math. 46 (1993), 27-56.

[36] Li, P; Schoen, R: Lp and mean value properties of subharmonic functions on Riemannian manifolds. Acta Math. 153 (1984), no. 3-4, 279-301.

[37] Mabuchi, T: Some symplectic geometry on compact Kähler manifolds. I, Osaka Journal of Mathematics 24 (1987), 227-252.

[38] Messer, J; Spohn, H: Statistical mechanics of the isothermal Lane-Emden equation. J. Statist. Phys. 29 (1982), no. 3, 561-578,

[39] Manin, Yu. I. New dimensions in geometry. Workshop Bonn 1984 (Bonn, 1984), 59-101. Lecture Notes in Math., 1111, Springer, Berlin, 1985.

[40] Jeffres, T; Mazzeo, R; Rubinstein, Y.A:; Kähler-Einstein metrics with edge singularities. Ann. of Math. (2) 183 (2016), no. 1, 95-176.

[41] Rubinstein, Y.A: Some discretizations of geometric evolution equations and the Ricci iteration on the space of K" ahler metrics, Adv. Math. 218 (2008), 1526-1565.

[42] Siu, Y-T: Finite Generation of Canonical Ring by Analytic Method. arXiv:0803.2454

[43] Streets, J: Long time existence of Minimizing Moveme nt solutions of Calabi flow, arXiv:1208.2718

[44] Song, Y; Tian, G: The Kahler-Ricci flow through singularities. arXiv preprint arXiv:0909.4898, 2009

[45] Song, Y; Tian, G: Canonical measures and Kähler-Ricci flow. J. Amer. Math. Soc. 25 (2012), no. 2, 303-353.

[46] Székelyhidi, G: Greatest lower bounds on the Ricci curvature of Fano manifolds. Compos. Math. 147 (2011), no. 1, 319-331

[47] Tian, G: K-stability and Kähler-Einstein metrics. Comm. Pure Appl. Math. 68 (2015), no. 7, 1085-1156.

[48] Tsuji,H: Canonical measures and the dynamical systems of Bergman kernels. Preprint arXiv:0805.1829, 2008

[49] Zabrodin, A; Matrix models and growth processes: from viscous flows to the quantum Hall effect. Preprint in 2004 at arXiv.org/abs/hep-th/0411437

[50] Zelditch, S: Large deviations of empirical measures of zeros on Riemann surfaces. Int Math Res Notices (2012)

[51] Yau, S-T: On the Ricci curvature of a compact K" ahler manifold and the complex MongeAmp'ere equation", Comm. Pure Appl. Math. 31 (1978) 